\title{
General Decay Rates for a Laminated Beam with Memory
}

\author{
Zhijing Chen, Wenjun Liu* and Dongqin Chen
}

\begin{abstract}
In previous work 23, Mustafa considered a viscoelastic laminated beam system with structural damping in the case of equal-speed wave propagations, and established explicit energy decay formula which gives the best decay rates. In this paper, we continue to consider the similar problems and establish the general decay result for the energy, to system with structural damping in the case of non-equal wave speeds and to system without structural damping in the case of equal wave speeds, respectively. For the first case, we use the second-order energy method to overcome the difficulty of estimating the non-equal speeds term. For the second case, we construct an appropriated perturbed functional to estimate $\left\|w_{t}\right\|_{2}^{2}$ so as to overcome the absence of structural damping.
\end{abstract}

\section{Introduction}

In previous work 23, Mustafa considered the following viscoelastic laminated beam system with structural damping

$$
\begin{cases}\rho \varphi_{t t}+G\left(\psi-\varphi_{x}\right)_{x}=0 & (x, t) \in(0,1) \times(0, \infty), \\ I_{\rho}(3 w-\psi)_{t t}-G\left(\psi-\varphi_{x}\right)-D(3 w-\psi)_{x x} & \\ \quad+\int_{0}^{t} g(t-s)(3 w-\psi)_{x x}(s) \mathrm{d} s=0 & (x, t) \in(0,1) \times(0, \infty), \\ I_{\rho} w_{t t}-D w_{x x}+G\left(\psi-\varphi_{x}\right)+\frac{4}{3} \gamma w+\frac{4}{3} \beta w_{t}=0 & (x, t) \in(0,1) \times(0, \infty)\end{cases}
$$

under initial conditions

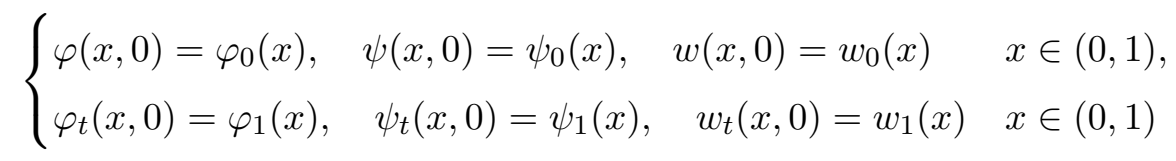

and boundary conditions

$$
\varphi_{x}(0, t)=\varphi(1, t)=\psi(0, t)=\psi_{x}(1, t)=w(0, t)=w_{x}(1, t)=0, \quad t \in(0, \infty),
$$

where $\varphi$ denotes the transverse displacement of the beam which departs from its equilibrium position, $\psi$ denotes the rotation angle, $w$ is proportional to the amount of slip along

Received August 8, 2018; Accepted November 11, 2018.

Communicated by Eiji Yanagida.

2010 Mathematics Subject Classification. 34B05, 35L05, 93C20, 93D20.

Key words and phrases. general stability, laminated beam, memory, energy method.

*Corresponding author. 
the interface at time $t$ and longitudinal spatial variable $x, 3 w-\psi$ represents the effective rotation angle. Moreover, the third equation of (1.1) describes the dynamics of the slip; $\rho, G, I_{\rho}, D, \gamma, \beta$ are positive constant coefficients and denote the density of the beams, the shear stiffness, the mass moment of inertia, the flexural rigidity, the adhesive stiffness of the beams, and the adhesive damping parameter, respectively. If $\beta \neq 0$, the adhesion at the interface supplies a restoring force proportion to the interfacial slip. If $\beta=0$, the third equation of (1.1) describes the coupled laminated beams without structural damping at the interface. In that paper, the author established the general decay result under the equal-speed wave propagation case: $G / \rho=D / I_{\rho}$. As for the previous results and developments of the viscoelastic laminated beam system, the authors have stated and summarized in great detail [23], thus we just omit it here. The readers, for a better understanding of present work, are recommended to see [1, 6, 10, 13, 16, 20, 25, 27, 29] and the references therein.

Apart from studying the laminated beam system itself, many people have been interested in the relationship between the laminated beam and the thermal conditions. For example, Liu and Zhao [18] considered the stabilization of a thermoelastic laminated beam with past history as

$$
\begin{cases}\rho \varphi_{t t}+G\left(\psi-\varphi_{x}\right)_{x}+\theta_{x}=0 & (x, t) \in(0,1) \times(0, \infty), \\ I_{\rho}(3 w-\psi)_{t t}-G\left(\psi-\varphi_{x}\right)-D(3 w-\psi)_{x x} & \\ \quad+\int_{0}^{\infty} g(s)(3 w-\psi)_{x x}(x, t-s) \mathrm{d} s-\theta=0 & (x, t) \in(0,1) \times(0, \infty), \\ I_{\rho} w_{t t}-D w_{x x}+G\left(\psi-\varphi_{x}\right)+\frac{4}{3} \gamma w+\frac{4}{3} \beta w_{t}=0 & (x, t) \in(0,1) \times(0, \infty), \\ k \theta_{t}-\tau \theta_{x x}+\varphi_{x t}+(3 w-\psi)_{t}=0 & (x, t) \in(0,1) \times(0, \infty),\end{cases}
$$

in which, $g$ is assumed to satisfy, for a positive constant $\xi$ and $1 \leq p \leq 3 / 2$,

$$
g^{\prime}(t) \leq-\xi g^{p}(t), \quad \forall t \leq 0 .
$$

For system (1.4) with structural damping and without any restriction on the speeds of wave propagations, they obtained the exponential and polynomial stabilities. For system (1.4) without structural damping, they established the exponential and polynomial stabilities in case of equal speeds and lack of exponential stability in case of non-equal speeds. In the recent work [20], Apalara investigated a laminated beam with structural damping under Cattaneo's law of heat conduction, and proved the exponential and polynomial stability results depend on a stability number. However, the case of the absence of structural damping was left as an open problem. We refer the reader to $3,5,7,9,14,15,17,19,24,28$ for some other related results.

In this paper, we first investigate the general decay rate of the solutions for problem $1.1-1.3$ with structural damping $(\beta \neq 0)$ in the case of non-equal wave speeds: 
$G / \rho \neq D / I_{\rho}$. For our this purpose, we use the second-order energy method to overcome the difficulty of estimating the non-equal speeds term. We then consider system (1.1)(1.3) without structural damping $(\beta=0)$, and prove the general stability in the case of equal wave speeds $G / \rho=D / I_{\rho}$ by using the perturbed energy method. To overcome the absence of structural damping $(\beta=0)$, we shall construct an appropriated perturbed functional $J(t)$ (see Lemma 5.1 below) to estimate $\left\|w_{t}\right\|_{2}^{2}$.

This paper is organized as follows. In Section 2, we give some assumptions. In Section 3. we state and prove some technical lemmas that are useful in the entire work. In Section 4, we state and prove decay result for system $1.1-1.3$ with structural damping in the case of non-equal wave speeds. Finally, Section 5 is devoted to proving the decay result for system (1.1)- 1.3 without structural damping in the case of equal wave speeds.

\section{Preliminaries and main results}

In this section, we give some assumptions and state some main theorems. We use $c>0$ to denote a positive constant which does not depend on the initial data. First, we consider the following assumptions as in 23$]$ :

(A1) $g: \mathbb{R}^{+} \rightarrow \mathbb{R}^{+}$is a non-increasing differentiable function such that

$$
g(0)>0, \quad D-\int_{0}^{+\infty} g(s) \mathrm{d} s=l>0 .
$$

(A2) There exists an non-increasing differentiable function $\xi: \mathbb{R}^{+} \rightarrow \mathbb{R}^{+}$and a $C^{1}$ function $H:[0, \infty) \rightarrow[0, \infty)$ which is either linear or strictly increasing and strictly convex $C^{2}$ function on $(0, r], r \leq g(0)$, with $H(0)=H^{\prime}(0)=0$, such that

$$
g^{\prime}(t) \leq-\xi(t) H(g(t)), \quad \forall t \geq 0 .
$$

Remark 2.1. 23, Remark 2.8] (1) From assumption (A1), we deduce that

$$
g(t) \rightarrow 0 \text { as } t \rightarrow+\infty \text { and } g(t) \leq \frac{D-l}{t}, \forall t>0
$$

Furthermore, from the assumption (A2), we obtain that there exists $t_{0}>0$ such that

$$
g\left(t_{0}\right)=r \quad \text { and } \quad g(t) \leq r, \quad \forall t \geq t_{0} .
$$

The non-increasing property of $g$ gives

$$
0<g\left(t_{0}\right) \leq g(t) \leq g(0), \quad \forall t \in\left[0, t_{0}\right]
$$

A combination of this with the continuity of $H$, for two constants $a, d>0$, yields

$$
a \leq H(g(t)) \leq d, \quad \forall t \in\left[0, t_{0}\right]
$$


Consequently, for any $t \in\left[0, t_{0}\right]$, we get

$$
g^{\prime}(t) \leq-\xi(t) H(g(t)) \leq-a \xi(t)=-\frac{a}{g(0)} \xi(t) g(0) \leq-\frac{a}{g(0)} \xi(t) g(t)
$$

and, hence,

$$
\xi(t) g(t) \leq-\frac{g(0)}{a} g^{\prime}(t), \quad \forall t \in\left[0, t_{0}\right]
$$

(2) If $H$ is a strictly increasing and strictly convex $C^{2}$ function on $(0, r]$, with $H(0)=$ $H^{\prime}(0)=0$, then it has an extension $\bar{H}$, which is a strictly increasing and strictly convex $C^{2}$ function on $(0, \infty)$. For example, if $H(r)=A, H^{\prime}(r)=B, H^{\prime \prime}(r)=C$, we can define $\bar{H}$, for any $t>r$, by

$$
\bar{H}(t)=\frac{C}{2} t^{2}+(B-C r) t+\left(A+\frac{C}{2} r^{2}-B r\right) .
$$

Next, we introduce the vector function

$$
U=\left(\varphi, 3 w-\psi, w, \varphi_{t}, 3 w_{t}-\psi_{t}, w_{t}\right)^{T}
$$

and

$$
U_{0}=\left(\varphi_{0}, 3 w_{0}-\psi_{0}, w_{0}, \varphi_{1}, 3 w_{1}-\psi_{1}, w_{1}\right)^{T} .
$$

Then, we consider the following Hilbert spaces

$$
\mathscr{H}_{0}=\widetilde{H}_{*}^{1}(0,1) \times\left(H_{*}^{1}(0,1)\right)^{2} \times\left(L^{2}(0,1)\right)^{3}
$$

and

$$
\begin{aligned}
& \mathscr{H}_{1}=\left\{U \in \mathscr{H}_{0} \mid \varphi \in \widetilde{H}_{*}^{2}(0,1), 3 w-\psi, w \in H_{*}^{2}(0,1), \varphi_{t} \in \widetilde{H}_{*}^{1}(0,1),\right. \\
&\left.3 w_{t}-\psi_{t}, w_{t} \in H_{*}^{1}(0,1), \varphi_{x}(0, t)=0, \psi_{x}(1, t)=w_{x}(1, t)=0\right\},
\end{aligned}
$$

where

$$
\begin{array}{cl}
H_{*}^{1}(0,1)=\left\{\eta \mid \eta \in H^{1}(0,1), \eta(0)=0\right\}, & \widetilde{H}_{*}^{1}(0,1)=\left\{\eta \mid \eta \in H^{1}(0,1), \eta(1)=0\right\}, \\
H_{*}^{2}(0,1)=H^{2}(0,1) \cap H_{*}^{1}(0,1), & \widetilde{H}_{*}^{2}(0,1)=H^{2}(0,1) \cap \widetilde{H}_{*}^{1}(0,1) .
\end{array}
$$

For completeness, we state, without proof, the following global existence and regularity result which can be easily proved by the standard Galerkin method.

Theorem 2.2. [13 Let $U_{0} \in \mathscr{H}_{0}$ be given. Assume that g satisfies hypothesis (A1). Then problem 1.1 - 1.3) has a unique weak solution

$$
\begin{gathered}
\varphi \in C\left(\mathbb{R}^{+} ; \widetilde{H}_{*}^{1}(0,1)\right) \cap C^{1}\left(\mathbb{R}^{+} ; L^{2}(0,1)\right), \\
3 w-\psi, w \in C\left(\mathbb{R}^{+} ; H_{*}^{1}(0,1)\right) \cap C^{1}\left(\mathbb{R}^{+} ; L^{2}(0,1)\right) .
\end{gathered}
$$


Moreover, if $U_{0} \in \mathscr{H}_{1}$, then the solution satisfies

$$
\begin{gathered}
\varphi \in C\left(\mathbb{R}^{+} ; \widetilde{H}_{*}^{2}(0,1)\right) \cap C^{1}\left(\mathbb{R}^{+} ; \widetilde{H}_{*}^{1}(0,1)\right) \cap C^{2}\left(\mathbb{R}^{+} ; L^{2}(0,1)\right), \\
3 w-\psi, w \in C\left(\mathbb{R}^{+} ; H_{*}^{2}(0,1)\right) \cap C^{1}\left(\mathbb{R}^{+} ; H_{*}^{1}(0,1)\right) \cap C^{2}\left(\mathbb{R}^{+} ; L^{2}(0,1)\right) .
\end{gathered}
$$

Now, we introduce the following energy functional:

$$
\begin{aligned}
E(t)= & \frac{1}{2}\left(\rho\left\|\varphi_{t}\right\|_{2}^{2}+I_{\rho}\left\|3 w_{t}-\psi_{t}\right\|_{2}^{2}+3 I_{\rho}\left\|w_{t}\right\|_{2}^{2}+G\left\|\psi-\varphi_{x}\right\|_{2}^{2}\right. \\
& \left.+\left(D-\int_{0}^{t} g(s) \mathrm{d} s\right)\left\|3 w_{x}-\psi_{x}\right\|_{2}^{2}+3 D\left\|w_{x}\right\|_{2}^{2}+4 \gamma\|w\|_{2}^{2}\right) \\
& +\frac{1}{2}\left(g \circ\left(3 w_{x}-\psi_{x}\right)\right)(t),
\end{aligned}
$$

where, for any $v \in L^{2}(0,1)$,

$$
(g \circ v)(t)=\int_{0}^{1} \int_{0}^{t} g(t-s)(v(t)-v(s))^{2} \mathrm{~d} s \mathrm{~d} x .
$$

The following lemmas play an important role in the proof of our main results.

Lemma 2.3. 13 The following inequalities hold:

$$
\begin{aligned}
& \left(\int_{0}^{t} g(t-s)\left(\left(3 w_{x}-\psi_{x}\right)(t)-\left(3 w_{x}-\psi_{x}\right)(s)\right) \mathrm{d} s\right)^{2} \\
\leq & g_{0}(t) \int_{0}^{t} g(t-s)\left(\left(3 w_{x}-\psi_{x}\right)(t)-\left(3 w_{x}-\psi_{x}\right)(s)\right)^{2} \mathrm{~d} s, \\
& \left(\int_{0}^{t} g^{\prime}(t-s)\left(\left(3 w_{x}-\psi_{x}\right)(t)-\left(3 w_{x}-\psi_{x}\right)(s)\right) \mathrm{d} s\right)^{2} \\
\leq & -g(0) \int_{0}^{t} g^{\prime}(t-s)\left(\left(3 w_{x}-\psi_{x}\right)(t)-\left(3 w_{x}-\psi_{x}\right)(s)\right)^{2} \mathrm{~d} s,
\end{aligned}
$$

where $g_{0}(t)=\int_{0}^{t} g(s) \mathrm{d} s$.

Lemma 2.4 (Jensen's inequality). Let $P:[c, d] \rightarrow \mathbb{R}$ be a convex function. Assume that the functions $f: \Omega \rightarrow[c, d]$ and $h: \Omega \rightarrow \mathbb{R}$ are integrable such that $h(x) \geq 0$ for any $x \in \Omega$ and $\int_{\Omega} h(x) \mathrm{d} x=k>0$. Then,

$$
P\left(\frac{1}{k} \int_{\Omega} f(x) h(x) \mathrm{d} x\right) \leq \frac{1}{k} \int_{\Omega} P(f(x)) h(x) \mathrm{d} x
$$

Lemma 2.5. 13 Let $(\varphi, 3 w-\psi, w)$ be the solution of $1.1-1.3$. Then

$$
\frac{d}{d t} E(t)=-4 \beta\left\|w_{t}\right\|_{2}^{2}-\frac{g(t)}{2}\left\|3 w_{x}-\psi_{x}\right\|_{2}^{2}+\frac{1}{2}\left(g^{\prime} \circ\left(3 w_{x}-\psi_{x}\right)\right)(t) \leq 0 .
$$


In [23, Mustafa presented the following general decay result for problem (1.1)-(1.3) with structural damping $(\beta \neq 0)$ in the case of equal wave speeds:

Theorem 2.6. 23 Let $U_{0} \in \mathscr{H}_{0}$. Assume that (A1) and (A2) hold and $G / \rho=D / I_{\rho}$. Then, there exist positive constants $k_{1}$ and $k_{2}$ such that the energy functional associated to problem (1.1) - (1.3) satisfies

$$
E(t) \leq k_{2} H_{1}^{-1}\left(k_{1} \int_{t_{0}}^{t} \xi(s) \mathrm{d} s\right), \quad \forall t>t_{0},
$$

where $H_{1}$ is given by $H_{1}(t)=\int_{t}^{r} \frac{1}{s H^{\prime}(s)} \mathrm{d} s$ and $t_{0}=g^{-1}(r)$.

We are now in a position to state our the first general decay result for problem (1.1) (1.3) with structural damping $(\beta \neq 0)$ in the case of non-equal wave speeds.

Theorem 2.7. Let $U_{0} \in \mathscr{H}_{0}$. Assume that (A1) and (A2) hold and $G / \rho \neq D / I_{\rho}$. Then, there exist positive constants $k_{1}, k_{2}$ and $t_{1}>t_{0}=g^{-1}(r)$ such that the energy functional associated to problem (1.1)-(1.3) satisfies the estimate

$$
E(t) \leq k_{2}\left(t-t_{0}\right) H_{2}^{-1}\left(\frac{k_{1}}{\left(t-t_{0}\right) \int_{t_{1}}^{t} \xi(s) \mathrm{d} s}\right), \quad \forall t>t_{1},
$$

where $\mathrm{H}_{2}$ is given by $H_{2}(t)=t H^{\prime}(t)$.

Remark 2.8. Assume $H(s)=s^{p}, 1 \leq p<2$ in (A2), then by simple calculations, we see that the decay rates of $E(t)$ is given by, for constants $C$ and $\bar{k}$,

$$
E(t) \leq \begin{cases}C\left(\frac{1+\int_{0}^{t} g(s) \mathrm{d} s}{\int_{0}^{t} \xi(s) \mathrm{d} s}\right) & \text { if } p=1, \\ \bar{k}\left(t-t_{0}\right)^{1-1 / p}\left(\frac{1}{\int_{0}^{t} \xi(s) \mathrm{d} s}\right)^{1 / p} & \text { if } 1<p<2 .\end{cases}
$$

Remark 2.9. Note that the estimate (2.2) was obtained by Li et al. in [13].

The second general decay result for problem (1.1)-(1.3) without structural damping $(\beta=0)$ in the case of equal wave speeds reads as follows.

Theorem 2.10. Let $U_{0} \in \mathscr{H}_{0}$. Assume that (A1) and (A2) hold, $\beta=0$ and $G / \rho=D / I_{\rho}$. Then, there exist positive constants $k_{1}$ and $k_{2}$ such that the energy functional associated to problem 1.1 -1.3 satisfies

$$
E(t) \leq k_{2} H_{1}^{-1}\left(k_{1} \int_{t_{0}}^{t} \xi(s) \mathrm{d} s\right), \quad \forall t>t_{0},
$$

where $H_{1}$ is given by $H_{1}(t)=\int_{t}^{r} \frac{1}{s H^{\prime}(s)} \mathrm{d} s$ and $t_{0}=g^{-1}(r)$. 
Remark 2.11. Assume that $H(s)=s^{p}, 1 \leq p<2$ in (A2), then by simple calculations, we see that the decay rates of $E(t)$ is given by, for constants $k_{1}, k_{2}$ and $k_{3}$,

$$
E(t) \leq \begin{cases}k_{1} \exp \left(-k_{2} \int_{0}^{t} \xi(s) \mathrm{d} s\right) & \text { if } p=1, \\ k_{3}\left(1+\int_{0}^{t} \xi(s) \mathrm{d} s\right)^{-1 /(p-1)} & \text { if } 1<p<2 .\end{cases}
$$

\section{Technical lemmas}

In this section, we establish several lemmas needed to prove our main results. For our purpose, we will adopt the functionals introduced in 23 with some modifications.

Lemma 3.1. Assume that (A1) and (A2) hold. Then, the functional $F_{1}(t)$ defined as

$$
F_{1}(t)=-\rho \int_{0}^{1} \varphi \varphi_{t} \mathrm{~d} x
$$

satisfies, for any $\varepsilon_{1}>0$,

$$
F_{1}^{\prime}(t) \leq-\rho\left\|\varphi_{t}\right\|_{2}^{2}+G \varepsilon_{1}\left\|\psi-\varphi_{x}\right\|_{2}^{2}+\frac{G}{2 \varepsilon_{1}}\left\|3 w_{x}-\psi_{x}\right\|_{2}^{2}+\frac{9 G}{2 \varepsilon_{1}}\left\|w_{x}\right\|_{2}^{2} .
$$

Proof. By differentiating $F_{1}(t)$ with respect to $t$, using (1.1) and integrating by parts, we obtain

$$
F_{1}^{\prime}(t)=-\rho\left\|\varphi_{t}\right\|_{2}^{2}-G \int_{0}^{1} \varphi_{x}\left(\psi-\varphi_{x}\right) \mathrm{d} x
$$

Note that

$$
-G \int_{0}^{1} \varphi_{x}\left(\psi-\varphi_{x}\right) \mathrm{d} x \leq G \varepsilon_{1}\left\|\psi-\varphi_{x}\right\|_{2}^{2}+\frac{G}{2 \varepsilon_{1}}\left\|3 w_{x}-\psi_{x}\right\|_{2}^{2}+\frac{9 G}{2 \varepsilon_{1}}\left\|w_{x}\right\|_{2}^{2} .
$$

This completes the proof.

Lemma 3.2. Under the conditions (A1) and (A2), the functional $F_{2}(t)$ defined by

$$
F_{2}(t)=I_{\rho} \int_{0}^{1}(3 w-\psi)\left(3 w_{t}-\psi_{t}\right) \mathrm{d} x
$$

satisfies, for any $\varepsilon_{2}>0$,

$$
\begin{aligned}
F_{2}^{\prime}(t) \leq & -\left(l-\varepsilon_{2}(G+1)\right)\left\|3 w_{x}-\psi_{x}\right\|_{2}^{2}+I_{\rho}\left\|3 w_{t}-\psi_{t}\right\|_{2}^{2} \\
& +\frac{G}{4 \varepsilon_{2}}\left\|\psi-\varphi_{x}\right\|_{2}^{2}+\frac{D-l}{4 \varepsilon_{2}}\left(g \circ\left(3 w_{x}-\psi_{x}\right)\right)(t) .
\end{aligned}
$$

Proof. It follows from (1.1) and integration by parts that

$$
\begin{aligned}
F_{2}^{\prime}(t)= & I_{\rho}\left\|3 w_{t}-\psi_{t}\right\|_{2}^{2}+G \int_{0}^{1}(3 w-\psi)\left(\psi-\varphi_{x}\right) \mathrm{d} x-D\left\|3 w_{x}-\psi_{x}\right\|_{2}^{2} \\
& -\int_{0}^{1}(3 w-\psi) \int_{0}^{t} g(t-s)(3 w-\psi)_{x x}(s) \mathrm{d} s \mathrm{~d} x .
\end{aligned}
$$

Using Young's inequality and Lemma 2.3 with $\varepsilon_{2}>0$, we obtain 3.2 . 
Lemma 3.3. Assume that (A1) and (A2) hold. Then, the functional $F_{3}(t)$ defined by

$$
F_{3}(t)=I_{\rho} \int_{0}^{1} w w_{t} \mathrm{~d} x
$$

satisfies the estimate

$$
\begin{aligned}
F_{3}^{\prime}(t) \leq & -\left(\frac{4}{3} \gamma-\varepsilon_{3}\left(G+\frac{4 \beta}{3}\right)\right)\|w\|_{2}^{2}-D\left\|w_{x}\right\|_{2}^{2} \\
& +\left(I_{\rho}+\frac{\beta}{3 \varepsilon_{3}}\right)\left\|w_{t}\right\|_{2}^{2}+\frac{G}{4 \varepsilon_{3}}\left\|\psi-\varphi_{x}\right\|_{2}^{2}
\end{aligned}
$$

where $\varepsilon_{3}>0$.

Proof. Differentiating $F_{3}(t)$ with respect to $t$, using the third equation of (1.1) and integrating by parts, we obtain

$$
F_{3}^{\prime}(t)=I_{\rho}\left\|w_{t}\right\|_{2}^{2}-G \int_{0}^{1} w\left(\psi-\varphi_{x}\right) \mathrm{d} x-\frac{4 \gamma}{3}\|w\|_{2}^{2}-\frac{4 \beta}{3} \int_{0}^{1} w w_{t} \mathrm{~d} x-D\left\|w_{x}\right\|^{2} .
$$

Next, it follows from Young's inequality that

$$
F_{3}^{\prime}(t) \leq-\left(\frac{4 \gamma}{3}-\varepsilon_{3}\left(G+\frac{4 \beta}{3}\right)\right)\|w\|_{2}^{2}-D\left\|w_{x}\right\|_{2}^{2}+\left(I_{\rho}+\frac{\beta}{3 \varepsilon_{3}}\right)\left\|w_{t}\right\|_{2}^{2}+\frac{G}{4 \varepsilon_{3}}\left\|\psi-\varphi_{x}\right\|_{2}^{2},
$$

which completes the proof of Lemma 3.3 .

Lemma 3.4. Assume that (A1) and (A2) hold, after fixing $\varepsilon_{4}>0$, the functional $F_{4}(t)$ defined by

$$
F_{4}(t)=-I_{\rho} \int_{0}^{1}\left(3 w_{t}-\psi_{t}\right) \int_{0}^{t} g(t-s)[(3 w-\psi)(t)-(3 w-\psi)(s)] \mathrm{d} s \mathrm{~d} x
$$

satisfies the estimate

$$
\begin{aligned}
F_{4}^{\prime}(t) \leq & -\frac{I_{\rho} g_{0}(t)}{2}\left\|3 w_{t}-\psi_{t}\right\|_{2}^{2}+\frac{G \varepsilon_{4}}{2}\left\|\psi-\varphi_{x}\right\|_{2}^{2}+\frac{D \varepsilon_{4}}{2}\left\|3 w_{x}-\psi_{x}\right\|_{2}^{2} \\
& -\frac{I_{\rho} g(0) \lambda_{0}}{2 g_{0}(t)}\left(g^{\prime} \circ\left(3 w_{x}-\psi_{x}\right)\right)(t) \\
& +(D-l)\left(\frac{G \lambda_{0}}{2 \varepsilon_{4}}+\frac{D}{2 \varepsilon_{4}}\right)\left(g \circ\left(3 w_{x}-\psi_{x}\right)\right)(t)
\end{aligned}
$$

where $g_{0}(t)=\int_{0}^{t} g(s) \mathrm{d} s$.

Proof. Taking the derivative of $F_{4}(t)$ with respect to $t$ and using the second equation of (1.1), we get

$$
F_{4}^{\prime}(t)=-G \int_{0}^{1}\left(\psi-\varphi_{x}\right) \int_{0}^{t} g(t-s)[(3 w-\psi)(t)-(3 w-\psi)(s)] \mathrm{d} s \mathrm{~d} x
$$




$$
\begin{aligned}
& +\left(D-\int_{0}^{t} g(s) \mathrm{d} s\right) \int_{0}^{1}(3 w-\psi)_{x} \int_{0}^{t} g(t-s)\left[(3 w-\psi)_{x}(t)-(3 w-\psi)_{x}(s)\right] \mathrm{d} s \mathrm{~d} x \\
& +\left\|\int_{0}^{t} g(t-s)\left[(3 w-\psi)_{x}(t)-(3 w-\psi)_{x}(s)\right] \mathrm{d} s\right\|_{2}^{2}-I_{\rho}\left(\int_{0}^{t} g(s) \mathrm{d} s\right)\left\|3 w_{t}-\psi_{t}\right\|_{2}^{2} \\
& -I_{\rho} \int_{0}^{1}\left(3 w_{t}-\psi_{t}\right) \int_{0}^{t} g^{\prime}(t-s)[(3 w-\psi)(t)-(3 w-\psi)(s)] \mathrm{d} s \mathrm{~d} x .
\end{aligned}
$$

Now, we estimate the terms on the right-hand side of the above equation.

Using Young's inequality, Poincaré's inequality $\|u\|_{2}^{2} \leq \lambda_{0}\left\|u_{x}\right\|_{2}^{2}$ with $\lambda_{0}>0$ and Lemma 2.3. we obtain, for $0<\varepsilon_{4}<1$,

$$
\begin{aligned}
& -G \int_{0}^{1}\left(\psi-\varphi_{x}\right) \int_{0}^{t} g(t-s)[(3 w-\psi)(t)-(3 w-\psi)(s)] \mathrm{d} s \mathrm{~d} x \\
\leq & \frac{G \varepsilon_{4}}{2}\left\|\psi-\varphi_{x}\right\|_{2}^{2}+\frac{G}{2 \varepsilon_{4}} \int_{0}^{1}\left(\int_{0}^{t} \sqrt{g(t-s)} \sqrt{g(t-s)}[(3 w-\psi)(t)-(3 w-\psi)(s)] \mathrm{d} s\right)^{2} \mathrm{~d} x \\
\leq & \frac{G \varepsilon_{4}}{2}\left\|\psi-\varphi_{x}\right\|_{2}^{2}+\frac{G g_{0}(t)}{2 \varepsilon_{4}}(g \circ(3 w-\psi))(t) \\
\leq & \frac{G \varepsilon_{4}}{2}\left\|\psi-\varphi_{x}\right\|_{2}^{2}+\frac{G(D-l) \lambda_{0}}{2 \varepsilon_{4}}\left(g \circ\left(3 w_{x}-\psi_{x}\right)\right)(t) .
\end{aligned}
$$

Also, we have, for $0<\varepsilon_{4}<1$,

$$
\begin{aligned}
& \left(D-\int_{0}^{t} g(s) \mathrm{d} s\right) \int_{0}^{1}(3 w-\psi)_{x} \int_{0}^{t} g(t-s)\left[(3 w-\psi)_{x}(t)-(3 w-\psi)_{x}(s)\right] \mathrm{d} s \mathrm{~d} x \\
\leq & \frac{\left(D-g_{0}(t)\right) \varepsilon_{4}}{2}\left\|3 w_{x}-\psi_{x}\right\|_{2}^{2}+\frac{\left(D-g_{0}(t)\right)(D-l)}{2 \varepsilon_{4}}\left(g \circ\left(3 w_{x}-\psi_{x}\right)\right)(t) \\
\leq & \frac{D \varepsilon_{4}}{2}\left\|3 w_{x}-\psi_{x}\right\|_{2}^{2}+\frac{D(D-l)}{2 \varepsilon_{4}}\left(g \circ\left(3 w_{x}-\psi_{x}\right)\right)(t)
\end{aligned}
$$

and

$$
\left\|\int_{0}^{t} g(t-s)\left[(3 w-\psi)_{x}(t)-(3 w-\psi)_{x}(s)\right] \mathrm{d} s\right\|_{2}^{2} \leq(D-l)\left(g \circ\left(3 w_{x}-\psi_{x}\right)\right)(t) .
$$

Exploiting Young's inequality, Poincaré's inequality and Lemma 2.3, we obtain, for any $0<\varepsilon_{4}<1$,

$$
\begin{aligned}
& -I_{\rho} \int_{0}^{1}\left(3 w_{t}-\psi_{t}\right) \int_{0}^{t} g^{\prime}(t-s)[(3 w-\psi)(t)-(3 w-\psi)(s)] \mathrm{d} s \mathrm{~d} x \\
\leq & \frac{I_{\rho} g_{0}(t)}{2}\left\|3 w_{t}-\psi_{t}\right\|_{2}^{2}+\frac{I_{\rho}}{2 g_{0}(t)}\left\|\int_{0}^{t} g^{\prime}(t-s)[(3 w-\psi)(t)-(3 w-\psi)(s)] \mathrm{d} s\right\|_{2}^{2} \\
\leq & \frac{I_{\rho} g_{0}(t)}{2}\left\|3 w_{t}-\psi_{t}\right\|_{2}^{2}-\frac{I_{\rho} g(0)}{2 g_{0}(t)}\left(g^{\prime} \circ(3 w-\psi)\right)(t) \\
\leq & \frac{I_{\rho} g_{0}(t)}{2}\left\|3 w_{t}-\psi_{t}\right\|_{2}^{2}-\frac{I_{\rho} g(0) \lambda_{0}}{2 g_{0}(t)}\left(g^{\prime} \circ\left(3 w_{x}-\psi_{x}\right)\right)(t) .
\end{aligned}
$$

A combination of all the above estimates gives the desired result. 
Lemma 3.5. Assume that (A1) and (A2) hold. Then for any $\varepsilon_{5}>0$, the functional $F_{5}(t)$ defined by

$$
\begin{aligned}
F_{5}(t)= & \frac{D \rho}{G} \int_{0}^{1} \varphi_{t}\left(3 w_{x}-\psi_{x}\right) \mathrm{d} x-I_{\rho} \int_{0}^{1}\left(3 w_{t}-\psi_{t}\right)\left(\psi-\varphi_{x}\right) \mathrm{d} x \\
& -\frac{\rho}{G} \int_{0}^{1} \varphi_{t} \int_{0}^{t} g(t-s)\left(3 w_{x}-\psi_{x}\right)(s) \mathrm{d} s \mathrm{~d} x
\end{aligned}
$$

satisfies the estimate

$$
\begin{aligned}
F_{5}^{\prime}(t) \leq & -G\left\|\psi-\varphi_{x}\right\|_{2}^{2}+\left(\frac{D \rho}{G}-I_{\rho}\right) \int_{0}^{1} \varphi_{t}\left(3 w_{x t}-\psi_{x t}\right) \mathrm{d} x \\
& +18 \varepsilon_{5} I_{\rho}\left\|w_{t}\right\|_{2}^{2}+I_{\rho}\left(2 \varepsilon_{5}+\frac{1}{4 \varepsilon_{5}}\right)\left\|3 w_{t}-\psi_{t}\right\|_{2}^{2} \\
& +\frac{\varepsilon_{5} \rho}{G}(1+g(0))\left\|\varphi_{t}\right\|_{2}^{2}+\frac{\rho g(t)}{4 \varepsilon_{5} G}\left\|3 w_{x}-\psi_{x}\right\|_{2}^{2}-\frac{\rho g(0)}{4 \varepsilon_{5} G}\left(g^{\prime} \circ\left(3 w_{x}-\psi_{x}\right)\right)(t) .
\end{aligned}
$$

Proof. Taking the derivative of $F_{5}(t)$ with respect to $t$ and using (1.1), we have

$$
\begin{aligned}
F_{5}^{\prime}(t)= & -D \int_{0}^{1}\left(\psi-\varphi_{x}\right)_{x}\left(3 w_{x}-\psi_{x}\right) \mathrm{d} x+\frac{D \rho}{G} \int_{0}^{1} \varphi_{t}\left(3 w_{x t}-\psi_{x t}\right) \mathrm{d} x \\
& -G \int_{0}^{1}\left(\psi-\varphi_{x}\right)^{2} \mathrm{~d} x-D \int_{0}^{1}\left(3 w_{x x}-\psi_{x x}\right)\left(\psi-\varphi_{x}\right) \mathrm{d} x \\
& +\int_{0}^{1}\left(\psi-\varphi_{x}\right) \int_{0}^{t} g(t-s)\left(3 w_{x x}-\psi_{x x}\right)(s) \mathrm{d} s \mathrm{~d} x-I_{\rho} \int_{0}^{1}\left(3 w_{t}-\psi_{t}\right)\left(\psi-\varphi_{x}\right)_{t} \mathrm{~d} x \\
& +\int_{0}^{1}\left(\psi-\varphi_{x}\right)_{x} \int_{0}^{t} g(t-s)\left(3 w_{x}-\psi_{x}\right)(s) \mathrm{d} s \mathrm{~d} x \\
& -\frac{\rho}{G} \int_{0}^{1} \varphi_{t} \int_{0}^{t} g^{\prime}(t-s)\left(3 w_{x}-\psi_{x}\right)(s) \mathrm{d} s \mathrm{~d} x-\frac{\rho g(0)}{G} \int_{0}^{1} \varphi_{t}\left(3 w_{x}-\psi_{x}\right)(t) \mathrm{d} x
\end{aligned}
$$

Next, making use of Young's inequality, Lemma 2.1 and integrating by parts, we deduce that (3.5) (see [13] for details). This completes the proof.

As in 22$]$, we use the functional

$$
K(t)=\int_{0}^{1} \int_{0}^{t} f(t-s)\left|\left(3 w_{x}-\psi_{x}\right)(s)\right|^{2} \mathrm{~d} s \mathrm{~d} x
$$

where $f(t)=\int_{t}^{\infty} g(s) \mathrm{d} s$.

Lemma 3.6. 22, Lemma 3.6] Under conditions (A1) and (A2), the functional $K(t)$ satisfies the estimate

$$
K^{\prime}(t) \leq-\frac{1}{2}\left(g \circ\left(3 w_{x}-\psi_{x}\right)\right)(t)+3(D-l)\left\|3 w_{x}-\psi_{x}\right\|_{2}^{2}
$$


Lemma 3.7. The functional $\mathcal{L}$ defined by

$$
\mathcal{L}(t):=N E(t)+F_{1}(t)+N_{2} F_{2}(t)+N_{3} F_{3}(t)+N_{4} F_{4}(t)+N_{5} F_{5}(t)
$$

satisfies, for a suitable choice of $N, N_{2}, N_{3}, N_{4}, N_{5} \geq 0$,

$$
\mathcal{L}(t) \sim E(t)
$$

and the estimate

$$
\begin{aligned}
\mathcal{L}^{\prime}(t) \leq & -c_{1}\left[\left\|\varphi_{t}\right\|_{2}^{2}+\left\|3 w_{t}-\psi_{t}\right\|_{2}^{2}+\left\|w_{t}\right\|_{2}^{2}+\left\|w_{x}\right\|_{2}^{2}+\|w\|_{2}^{2}+\left\|\psi-\varphi_{x}\right\|_{2}^{2}+\left\|3 w_{x}-\psi_{x}\right\|_{2}^{2}\right] \\
& +c_{2}\left(g \circ\left(3 w_{x}-\psi_{x}\right)\right)(t)+N_{5}\left(\frac{D \rho}{G}-I_{\rho}\right) \int_{0}^{1} \varphi_{t}\left(3 w_{x t}-\psi_{x t}\right) \mathrm{d} x, \quad \forall t \geq t_{0},
\end{aligned}
$$

where $c_{1}>0, c_{2}>0$ and $t_{0}$ has been introduced in Remark 2.1.

Proof. Let $g_{0}(t)=\int_{0}^{t} g(s) \mathrm{d} s \geq \int_{0}^{t_{0}} g(s) \mathrm{d} s=g_{1} \geq 0$ for any $t \geq t_{0}$. According to 3.1 (3.5), we have

$$
\begin{aligned}
\mathcal{L}^{\prime}(t) \leq & -I_{\rho}\left(\frac{N_{4} g_{1}}{2}-N_{2}-N_{5}\left(2 \varepsilon_{5}+\frac{1}{4 \varepsilon_{5}}\right)\right)\left\|3 w_{t}-\psi_{t}\right\|_{2}^{2}-\left(\rho-\frac{N_{5} \varepsilon_{5} \rho}{G}(1+g(0))\right)\left\|\varphi_{t}\right\|_{2}^{2} \\
& -\left(4 N \beta-N_{3}\left(I_{\rho}+\frac{\beta}{3 \varepsilon_{3}}\right)-18 N_{5} \varepsilon_{5} I_{\rho}\right)\left\|w_{t}\right\|_{2}^{2}-\left(N_{3}\left(\frac{4 \gamma}{3}-G \varepsilon_{3}\right)-N_{3} \frac{4 \beta \varepsilon_{3}}{3}\right)\|w\|_{2}^{2} \\
& -\left(N_{5} G-G \varepsilon_{1}-\frac{G N_{2}}{4 \varepsilon_{2}}-\frac{N_{4} G \varepsilon_{4}}{2}-\frac{N_{3} G}{4 \varepsilon_{3}}\right)\left\|\psi-\varphi_{x}\right\|_{2}^{2}-\left(N_{3} D-\frac{9 G}{2 \varepsilon_{1}}\right)\left\|w_{x}\right\|_{2}^{2} \\
& -\left(g(t)\left(\frac{N}{2}-\frac{N_{5} \rho}{4 \varepsilon_{5} G}\right)+N_{2}\left(l-\varepsilon_{2}(G+1)\right)-\frac{G}{2 \varepsilon_{1}}-\frac{N_{4} D \varepsilon_{4}}{2}\right)\left\|3 w_{x}-\psi_{x}\right\|_{2}^{2} \\
& +(D-l)\left(\frac{N_{2}}{4 \varepsilon_{2}}+N_{4}\left(\frac{G \lambda_{0}}{2 \varepsilon_{4}}+\frac{D}{2 \varepsilon_{4}}\right)\right)\left(g \circ\left(3 w_{x}-\psi_{x}\right)\right)(t) \\
& +\left(\frac{N}{2}-\frac{N_{4} I_{\rho} g(0) \lambda_{0}}{2 g_{0}(t)}-\frac{N_{5} \rho g(0)}{4 \varepsilon_{5} G}\right)\left(g^{\prime} \circ\left(3 w_{x}-\psi_{x}\right)\right)(t) \\
& +N_{5}\left(\frac{D \rho}{G}-I_{\rho}\right) \int_{0}^{1} \varphi_{t}\left(3 w_{x t}-\psi_{x t}\right) \mathrm{d} x .
\end{aligned}
$$

At this point, we need to choose our constants very carefully. First, we choose

$$
\varepsilon_{1}=G, \quad \varepsilon_{2}=\frac{1}{N_{2}}, \quad \varepsilon_{3}=\frac{1}{N_{3}}, \quad \varepsilon_{4}=\frac{1}{N_{4}}, \quad \varepsilon_{5}=\frac{G}{2 N_{5}(1+g(0))},
$$

and (3.7) becomes

$$
\begin{aligned}
\mathcal{L}^{\prime}(t) \leq & -\frac{\rho}{2}\left\|\varphi_{t}\right\|_{2}^{2}-I_{\rho}\left(\frac{N_{4} g_{1}}{2}-N_{2}-\frac{N_{5}^{2}(1+g(0))}{2 G}-\frac{G}{1+g(0)}\right)\left\|3 w_{t}-\psi_{t}\right\|_{2}^{2} \\
& -\left(4 N \beta-N_{3}\left(I_{\rho}+\frac{N_{3} \beta}{3}\right)-\frac{9 I_{\rho} G}{1+g(0)}\right)\left\|w_{t}\right\|_{2}^{2}-\left(\frac{4 N_{3} \gamma}{3}-\left(G+\frac{4 \beta}{3}\right)\right)\|w\|_{2}^{2} \\
& -\left(N_{5} G-\frac{G N_{2}^{2}}{4}-\frac{G N_{3}^{2}}{4}-\frac{G}{2}-G^{2}\right)\left\|\psi-\varphi_{x}\right\|_{2}^{2}-\left(N_{3} D-\frac{9}{2}\right)\left\|w_{x}\right\|_{2}^{2}
\end{aligned}
$$




$$
\begin{aligned}
& -\left(g(t)\left(\frac{N}{2}-\frac{N_{5}^{2} \rho(1+g(0))}{2 G^{2}}\right)+N_{2} l-\left(\frac{3}{2}+G+\frac{D}{2}\right)\right)\left\|3 w_{x}-\psi_{x}\right\|_{2}^{2} \\
& +(D-l)\left(\frac{N_{2}^{2}}{4}+N_{4}^{2}\left(\frac{G \lambda_{0}}{2}+\frac{D}{2}\right)\right)\left(g \circ\left(3 w_{x}-\psi_{x}\right)\right)(t) \\
& +\left(\frac{N}{2}-N_{4} \frac{I_{\rho} g(0) \lambda_{0}}{2 g_{0}(t)}-\frac{N_{5}^{2} \rho g(0)(1+g(0))}{2 G^{2}}\right)\left(g^{\prime} \circ\left(3 w_{x}-\psi_{x}\right)\right)(t) \\
& +N_{5}\left(\frac{D \rho}{G}-I_{\rho}\right) \int_{0}^{1} \varphi_{t}\left(3 w_{x t}-\psi_{x t}\right) \mathrm{d} x .
\end{aligned}
$$

Then, we select $N_{2}$ large enough so that

$$
N_{2} l-\left(\frac{3}{2}+G+\frac{D}{2}\right)>0 .
$$

Next, we choose $N_{3}$ large enough so that

$$
N_{3} D-\frac{9}{2}>0 \text { and } \frac{4 N_{3} \gamma}{3}-\left(G+\frac{4 \beta}{3}\right)>0 .
$$

Furthermore, we select $N_{5}$ large enough so that

$$
N_{5} G-G \frac{N_{2}^{2}}{4}-G \frac{N_{3}^{2}}{4}-\frac{G}{2}-G^{2}>0 .
$$

After that, we choose $N_{4}$ large enough so that

$$
\frac{N_{4} g_{1}}{2}-N_{2}-\frac{N_{5}^{2}(1+g(0))}{2 G}-\frac{G}{1+g(0)}>0 .
$$

Finally, let us choose $N$ large enough so that

$$
4 N \beta-N_{3}\left(I_{\rho}+\frac{N_{3} \beta}{3}\right)-\frac{9 I_{\rho} G}{1+g(0)}>0, \quad \frac{N}{2}-\frac{N_{5}^{2} \rho(1+g(0))}{2 G^{2}}>0,
$$

and

$$
\frac{N}{2}-\frac{N_{4} I_{\rho} g(0) \lambda_{0}}{2 g_{0}(t)}-\frac{N_{5}^{2} \rho g(0)(1+g(0))}{2 G^{2}}>0 .
$$

So we arrive at, for positive constants $c_{1}$ and $c_{2}$,

$$
\begin{aligned}
\mathcal{L}^{\prime}(t) \leq & -c_{1}\left[\left\|\varphi_{t}\right\|_{2}^{2}+\left\|3 w_{t}-\psi_{t}\right\|_{2}^{2}+\left\|w_{t}\right\|_{2}^{2}+\left\|w_{x}\right\|_{2}^{2}+\|w\|_{2}^{2}+\left\|\psi-\varphi_{x}\right\|_{2}^{2}+\left\|3 w_{x}-\psi_{x}\right\|_{2}^{2}\right] \\
& +c_{2}\left(g \circ\left(3 w_{x}-\psi_{x}\right)\right)(t)+N_{5}\left(\frac{D \rho}{G}-I_{\rho}\right) \int_{0}^{1} \varphi_{t}\left(3 w_{x t}-\psi_{x t}\right) \mathrm{d} x .
\end{aligned}
$$

On the other hand, we find that

$$
\begin{aligned}
|\mathcal{L}(t)-N E(t)| \leq & \rho \int_{0}^{1}\left|\varphi \varphi_{t}\right| \mathrm{d} x+N_{2} I_{\rho} \int_{0}^{1}\left|(3 w-\psi)\left(3 w_{t}-\psi_{t}\right)\right| \mathrm{d} x+N_{3} I_{\rho} \int_{0}^{1}\left|w w_{t}\right| \mathrm{d} x \\
& +N_{4} I_{\rho} \int_{0}^{1}\left|3 w_{t}-\psi_{t}\right| \int_{0}^{t} g(t-s)|(3 w-\psi)(t)-(3 w-\psi)(s)| \mathrm{d} s \mathrm{~d} x
\end{aligned}
$$




$$
\begin{aligned}
& +N_{5}\left[\frac{D \rho}{G} \int_{0}^{1}\left|\varphi_{t}\left(3 w_{x}-\psi_{x}\right)\right| \mathrm{d} x+I_{\rho} \int_{0}^{1}\left|\left(3 w_{t}-\psi_{t}\right)\left(\psi-\varphi_{x}\right)\right| \mathrm{d} x\right. \\
& \left.\quad+\frac{\rho}{G} \int_{0}^{1}\left|\varphi_{t}\right| \int_{0}^{t} g(t-s)\left|\left(3 w_{x}-\psi_{x}\right)(s)\right| \mathrm{d} s \mathrm{~d} x\right] \\
& \leq c E(t),
\end{aligned}
$$

where $c$ is a positive constant.

Therefore, we can choose $N$ even large (if needed) so that the proof is completed.

4. The case with structural damping under non-equal wave speeds

In this section, we will give an estimate to the decay rate for system with structural damping in the case of non-equal speeds of wave propagation. We give some lemmas that is beneficial to prove the main result.

We introduce the second-order energy as

$$
\begin{aligned}
\widetilde{E}(t)=\frac{1}{2} & \left(\rho\left\|\varphi_{t t}\right\|_{2}^{2}+I_{\rho}\left\|3 w_{t t}-\psi_{t t}\right\|_{2}^{2}+3 I_{\rho}\left\|w_{t t}\right\|_{2}^{2}+G\left\|\psi_{t}-\varphi_{x t}\right\|_{2}^{2}+3 D\left\|w_{x t}\right\|_{2}^{2}+4 \gamma\left\|w_{t}\right\|_{2}^{2}\right. \\
& \left.+\left(D-\int_{0}^{t} g(s) \mathrm{d} s\right)\left\|3 w_{x t}-\psi_{x t}\right\|_{2}^{2}\right)+\frac{1}{2}\left(g \circ\left(3 w_{x t}-\psi_{x t}\right)\right)(t) .
\end{aligned}
$$

Then we give the following lemmas.

Lemma 4.1. [13] Let $U$ be the strong solution of $11.1-1.3$. Then, the second energy functional satisfies, for all $t \geq 0$,

$$
\begin{aligned}
\widetilde{E}^{\prime}(t)= & -4 \beta\left\|w_{t t}\right\|_{2}^{2}-\frac{g(t)}{2}\left\|3 w_{x t}-\psi_{x t}\right\|_{2}^{2}+\frac{1}{2}\left(g^{\prime} \circ\left(3 w_{x t}-\psi_{x t}\right)\right)(t) \\
& -g(t) \int_{0}^{1}\left(3 w_{t t}-\psi_{t t}\right)\left(3 w_{0 x x}-\psi_{0 x x}\right) \mathrm{d} x
\end{aligned}
$$

and

$$
\widetilde{E}(t) \leq c\left(\widetilde{E}(0)+\left\|3 w_{0 x x}-\psi_{0 x x}\right\|_{2}^{2}\right)
$$

Lemma 4.2. 13 Let $U$ be the strong solution of $(1.1)-(1.3)$. Then, we have

$$
\begin{aligned}
& \left(\frac{D \rho}{G}-I_{\rho}\right) \int_{0}^{1} \varphi_{t}\left(3 w_{x t}-\psi_{x t}\right) \mathrm{d} x \\
\leq & \varepsilon\left\|\varphi_{t}\right\|_{2}^{2}+\frac{c}{\varepsilon} g(t) E(0)+\frac{c}{\varepsilon}\left(g \circ\left(3 w_{x t}-\psi_{x t}\right)-g^{\prime} \circ\left(3 w_{x t}-\psi_{x t}\right)\right)(t),
\end{aligned}
$$

where $c>0$ and $\varepsilon>0$.

As in [21], we give the following inequality which is important to prove our main result. 
Lemma 4.3. Let $U$ be the strong solution of (1.1)-1.3. Assume that conditions (A1) and (A2) hold with $H$ being linear. Then

$$
\xi(t)\left(g \circ\left(3 w_{x t}-\psi_{x t}\right)\right)(t) \leq c\left(-\widetilde{E}^{\prime}(t)+C_{1} g(t)\right), \quad \forall t \geq 0
$$

for some positive constants $c$ and $C_{1}$.

Proof. Using the non-increasing property of $\xi(t)$ and $H$ is linear, we obtain

$$
\begin{aligned}
& \xi(t)\left(g \circ\left(3 w_{x t}-\psi_{x t}\right)\right)(t) \\
\leq & \int_{0}^{1} \int_{0}^{t} \xi(t-s) g(t-s)\left[\left(3 w_{x t}-\psi_{x t}\right)(t)-\left(3 w_{x t}-\psi_{x t}\right)(s)\right]^{2} \mathrm{~d} s \mathrm{~d} x \\
\leq & \int_{0}^{1} \int_{0}^{t} g^{\prime}(t-s)\left[\left(3 w_{x t}-\psi_{x t}\right)(t)-\left(3 w_{x t}-\psi_{x t}\right)(s)\right]^{2} \mathrm{~d} s \mathrm{~d} x \\
= & -\left(g^{\prime} \circ\left(3 w_{x t}-\psi_{x t}\right)\right)(t), \quad \forall t>0 .
\end{aligned}
$$

From equation (4.1), inequality 4.2 and Young inequality, we have, for any $t \geq 0$,

$$
\begin{aligned}
-\left(g^{\prime} \circ\left(3 w_{x t}-\psi_{x t}\right)\right)(t)= & -2 \widetilde{E}^{\prime}(t)-8 \beta\left\|w_{t t}\right\|_{2}^{2}-g(t)\left\|3 w_{x t}-\psi_{x t}\right\|_{2}^{2} \\
& -2 g(t) \int_{0}^{1}\left(3 w_{t t}-\psi_{t t}\right)\left(3 w_{0 x x}-\psi_{0 x x}\right) \mathrm{d} x \\
\leq & -2 \widetilde{E}^{\prime}(t)-2 g(t) \int_{0}^{1}\left(3 w_{t t}-\psi_{t t}\right)\left(3 w_{0 x x}-\psi_{0 x x}\right) \mathrm{d} x \\
\leq & -2 \widetilde{E}^{\prime}(t)+g(t)\left[\left\|3 w_{t t}-\psi_{t t}\right\|_{2}^{2}+\left\|3 w_{0 x x}-\psi_{0 x x}\right\|_{2}^{2}\right] \\
\leq & -2 \widetilde{E}^{\prime}(t)+g(t)\left(\frac{2 \widetilde{E}(t)}{I_{\rho}}+\left\|3 w_{0 x x}-\psi_{0 x x}\right\|_{2}^{2}\right) \\
\leq & c\left(-\widetilde{E}^{\prime}(t)+c_{1} g(t)\right)
\end{aligned}
$$

where $c$ and $C_{1}$ are some fixed positive constants.

Combining (4.3) and 4.4), we obtain the desired result.

Now, we turn to prove Theorem 2.7.

Proof of Theorem 2.7. Our proof starts with the observation that, for any $t \geq t_{0}$ and $m>0$,

$$
\begin{aligned}
\mathcal{L}^{\prime}(t) \leq & -c_{1}\left[\left\|\varphi_{t}\right\|_{2}^{2}+\left\|3 w_{t}-\psi_{t}\right\|_{2}^{2}+\left\|w_{t}\right\|_{2}^{2}+\left\|w_{x}\right\|_{2}^{2}+\|w\|_{2}^{2}+\left\|\psi-\varphi_{x}\right\|_{2}^{2}+\left\|3 w_{x}-\psi_{x}\right\|_{2}^{2}\right] \\
& +c_{2}\left(g \circ\left(3 w_{x}-\psi_{x}\right)\right)(t)+N_{5}\left(\frac{D \rho}{G}-I_{\rho}\right) \int_{0}^{1} \varphi_{t}\left(3 w_{x t}-\psi_{x t}\right) \mathrm{d} x \\
\leq & -m E(t)+c_{2}\left(g \circ\left(3 w_{x}-\psi_{x}\right)\right)(t)+N_{5}\left(\frac{D \rho}{G}-I_{\rho}\right) \int_{0}^{1} \varphi_{t}\left(3 w_{x t}-\psi_{x t}\right) \mathrm{d} x \\
\leq & -(m-\varepsilon) E(t)+c_{2}\left(g \circ\left(3 w_{x}-\psi_{x}\right)\right)(t) \\
& +\frac{c}{\varepsilon} g(t) E(0)+\frac{c}{\varepsilon}\left(g \circ\left(3 w_{x t}-\psi_{x t}\right)\right)-\left(g^{\prime} \circ\left(3 w_{x}-\psi_{x}\right)\right)(t),
\end{aligned}
$$


which are derived from $(3.6)$ and Lemma 4.2 . After fixing $\varepsilon$ small enough, we arrive at

$$
\mathcal{L}^{\prime}(t) \leq-m_{1} E(t)+c\left(g \circ\left(3 w_{x}-\psi_{x}\right)+g \circ\left(3 w_{x t}-\psi_{x t}\right)\right)(t)+c g(t) E(0)-c E^{\prime}(t),
$$

where $m_{1}$ is a fixed positive constant. Taking $\mathcal{F}(t):=\mathcal{L}(t)+c E(t)$, which is obviously equivalent to $E(t)$, to get, for any $t \geq t_{0}$,

$$
\mathcal{F}^{\prime}(t) \leq-m_{1} E(t)+c\left(g \circ\left(3 w_{x}-\psi_{x}\right)+g \circ\left(3 w_{x t}-\psi_{x t}\right)\right)(t)+c g(t)
$$

We consider the following two cases relying on the ideas present in [22].

Case 1: $H$ is linear. We multiply 4.5 by $\xi(t)$, then on account of assumption (A2), Lemma 4.3 and estimate 4.2 , we obtain, for any $t \geq t_{0}$,

$$
\xi(t) \mathcal{F}^{\prime}(t) \leq-m_{1} \xi(t) E(t)-c E^{\prime}(t)+c\left(-\widetilde{E}^{\prime}(t)+C_{1} g(t)\right)+c \xi(0) g(t) .
$$

As $\xi(t)$ is non-increasing, we have, for some fixed positive constant $c_{3}$,

$$
(\xi \mathcal{F}+c E+c \widetilde{E})^{\prime}(t) \leq-m_{1} \xi(t) E(t)+c_{3} g(t), \quad \forall t \geq t_{0} .
$$

It follows immediately that

$$
m_{1} \xi(t) E(t) \leq-(\xi \mathcal{F}+c E+c \widetilde{E})^{\prime}(t)+c_{3} g(t), \quad \forall t \geq t_{0} .
$$

According to the non-increasing property of $E(t)$ and estimate 4.2 , we may now integrate over $\left(t, t_{0}\right)$ to conclude that, for any $t>t_{0}$,

$$
\begin{aligned}
m_{1} E(t) \int_{t_{0}}^{t} \xi(s) \mathrm{d} s & \leq-(\xi \mathcal{F}+c E+c \widetilde{E})(t)+(\xi \mathcal{F}+c E+c \widetilde{E})\left(t_{0}\right)+c_{3} \int_{0}^{t} g(s) \mathrm{d} s \\
& \leq(\xi \mathcal{F}+c E+c \widetilde{E})(0)+c \int_{0}^{1}\left(3 w_{0 x x}-\psi_{0 x x}\right)^{2} \mathrm{~d} x+c_{3}(D-l) .
\end{aligned}
$$

Thus, we have, for some fixed positive constant $c$,

$$
E(t) \leq \frac{c}{\int_{t_{0}}^{t} \xi(s) \mathrm{d} s}, \quad \forall t>t_{0} .
$$

Case 2: $H$ is nonlinear. Taking account of (2.1), Lemma 2.5 and estimate (4.4), we get, for any $t \geq t_{0}$ and $c_{4}>0$,

$$
\begin{aligned}
& \int_{0}^{1} \int_{0}^{t_{0}} g(s)\left[\left(3 w_{x}-\psi_{x}\right)(t)-\left(3 w_{x}-\psi_{x}\right)(t-s)\right]^{2} \mathrm{~d} s \mathrm{~d} x \\
& +\int_{0}^{1} \int_{0}^{t_{0}} g(s)\left[\left(3 w_{x t}-\psi_{x t}\right)(t)-\left(3 w_{x t}-\psi_{x t}\right)(t-s)\right]^{2} \mathrm{~d} s \mathrm{~d} x \\
\leq & \frac{1}{\xi\left(t_{0}\right)} \int_{0}^{1} \int_{0}^{t_{0}} \xi(s) g(s)\left[\left(3 w_{x}-\psi_{x}\right)(t)-\left(3 w_{x}-\psi_{x}\right)(t-s)\right]^{2} \mathrm{~d} s \mathrm{~d} x
\end{aligned}
$$




$$
\begin{aligned}
& +\frac{1}{\xi\left(t_{0}\right)} \int_{0}^{1} \int_{0}^{t_{0}} \xi(s) g(s)\left[\left(3 w_{x t}-\psi_{x t}\right)(t)-\left(3 w_{x t}-\psi_{x t}\right)(t-s)\right]^{2} \mathrm{~d} s \mathrm{~d} x \\
\leq & -\frac{g(0)}{a \xi\left(t_{0}\right)} \int_{0}^{1} \int_{0}^{t_{0}} g^{\prime}(s)\left[\left(3 w_{x}-\psi_{x}\right)(t)-\left(3 w_{x}-\psi_{x}\right)(t-s)\right]^{2} \mathrm{~d} s \mathrm{~d} x \\
& +\frac{g(0)}{a \xi\left(t_{0}\right)} \int_{0}^{1} \int_{0}^{t_{0}} g^{\prime}(s)\left[\left(3 w_{x t}-\psi_{x t}\right)(t)-\left(3 w_{x t}-\psi_{x t}\right)(t-s)\right]^{2} \mathrm{~d} s \mathrm{~d} x \\
\leq & -c\left(E^{\prime}(t)+\widetilde{E}^{\prime}(t)\right)+c_{4} g(t) .
\end{aligned}
$$

Inserting this estimate into 4.5, we obtain, for any $t \geq t_{0}$ and $c_{5}>0$,

$$
\begin{aligned}
\mathcal{F}^{\prime}(t) \leq & -m_{1} E(t)-c\left(E^{\prime}(t)+\widetilde{E}^{\prime}(t)\right)+c_{5} g(t) \\
& +c \int_{t_{0}}^{t} g(s)\left\|\left(3 w_{x}-\psi_{x}\right)(t)-\left(3 w_{x}-\psi_{x}\right)(t-s)\right\|_{2}^{2} \mathrm{~d} s \\
& +c \int_{t_{0}}^{t} g(s)\left\|\left(3 w_{x t}-\psi_{x t}\right)(t)-\left(3 w_{x t}-\psi_{x t}\right)(t-s)\right\|_{2}^{2} \mathrm{~d} s .
\end{aligned}
$$

Now, we define the functional $\theta(t)$ by

$$
\begin{aligned}
\theta(t):=-\int_{t_{0}}^{t} g^{\prime}(s) & \left(\left\|\left(3 w_{x}-\psi_{x}\right)(t)-\left(3 w_{x}-\psi_{x}\right)(t-s)\right\|_{2}^{2}\right. \\
& \left.+\left\|\left(3 w_{x t}-\psi_{x t}\right)(t)-\left(3 w_{x t}-\psi_{x t}\right)(t-s)\right\|_{2}^{2}\right) \mathrm{d} s .
\end{aligned}
$$

Clearly, we have

$$
\begin{aligned}
& \theta(t) \leq-\int_{0}^{t} g^{\prime}(s)(\left\|\left(3 w_{x}-\psi_{x}\right)(t)-\left(3 w_{x}-\psi_{x}\right)(t-s)\right\|_{2}^{2} \\
&\left.+\left\|\left(3 w_{x t}-\psi_{x t}\right)(t)-\left(3 w_{x t}-\psi_{x t}\right)(t-s)\right\|_{2}^{2}\right) \mathrm{d} s \\
& \leq-c\left(E^{\prime}(t)+\widetilde{E}^{\prime}(t)\right)+c_{6} g(t), \quad \forall t>t_{0},
\end{aligned}
$$

where $c_{6}>0$ is a fixed constant.

After that, we define another functional $\eta(t)$ by, for any $t>t_{0}$,

$$
\begin{aligned}
\eta(t):=\frac{\gamma}{t-t_{0}} \int_{t_{0}}^{t}( & \left\|\left(3 w_{x}-\psi_{x}\right)(t)-\left(3 w_{x}-\psi_{x}\right)(t-s)\right\|_{2}^{2} \\
& \left.+\left\|\left(3 w_{x t}-\psi_{x t}\right)(t)-\left(3 w_{x t}-\psi_{x t}\right)(t-s)\right\|_{2}^{2}\right) \mathrm{d} s .
\end{aligned}
$$

Now, the following inequality holds under energy functional $E(t)$, second energy functional $\widetilde{E}(t)$, (4.1), Lemmas 2.5 and 4.1 that

$$
\begin{aligned}
\frac{1}{t-t_{0}} \int_{t_{0}}^{t} & \left(\left\|\left(3 w_{x}-\psi_{x}\right)(t)-\left(3 w_{x}-\psi_{x}\right)(t-s)\right\|_{2}^{2}\right. \\
& \left.+\left\|\left(3 w_{x t}-\psi_{x t}\right)(t)-\left(3 w_{x t}-\psi_{x t}\right)(t-s)\right\|_{2}^{2}\right) \mathrm{d} s
\end{aligned}
$$




$$
\begin{aligned}
& \leq \frac{4}{l\left(t-t_{0}\right)} \int_{t_{0}}^{t}(E(t)+E(t-s)+\widetilde{E}(t)+\widetilde{E}(t-s)) \mathrm{d} s \\
& \leq \frac{8}{l\left(t-t_{0}\right)} \int_{t_{0}}^{t}\left[E(0)+c\left(\widetilde{E}(0)+\int_{0}^{1}\left(3 w_{0 x x}-\psi_{0 x x}\right) \mathrm{d} x\right)\right] \mathrm{d} s \\
& \leq \frac{8}{l}\left[E(0)+c\left(\widetilde{E}(0)+\int_{0}^{1}\left(3 w_{0 x x}-\psi_{0 x x}\right) \mathrm{d} x\right)\right]<+\infty, \quad \forall t>t_{0} .
\end{aligned}
$$

Then the above inequality allows for a constant $0<\gamma<1$ chosen so that, for all $t>t_{0}$,

$$
0<\eta(t)<1
$$

otherwise we get the following decay rate from 4.6

$$
E(t) \leq \frac{c}{t-t_{0}}, \quad \forall t>t_{0}
$$

Moreover, recalling that $H$ is strict convex on $(0, r]$ and $H(0)=0$, then

$$
H(s \tau) \leq s H(\tau) \text { for } 0 \leq s \leq 1 \text { and } \tau \in(0, r]
$$

From assumption (A2), Lemma 2.4 and (4.6), it follows that, for any $t>t_{0}$,

$$
\begin{aligned}
& \theta(t)=-\int_{t_{0}}^{t} g^{\prime}(s)\left(\left\|\left(3 w_{x}-\psi_{x}\right)(t)-\left(3 w_{x}-\psi_{x}\right)(t-s)\right\|_{2}^{2}\right. \\
& \left.+\left\|\left(3 w_{x t}-\psi_{x t}\right)(t)-\left(3 w_{x t}-\psi_{x t}\right)(t-s)\right\|_{2}^{2}\right) \mathrm{d} s \\
& =-\frac{1}{\eta(t)} \int_{t_{0}}^{t} \eta(t) g^{\prime}(s)\left(\left\|\left(3 w_{x}-\psi_{x}\right)(t)-\left(3 w_{x}-\psi_{x}\right)(t-s)\right\|_{2}^{2}\right. \\
& \left.+\left\|\left(3 w_{x t}-\psi_{x t}\right)(t)-\left(3 w_{x t}-\psi_{x t}\right)(t-s)\right\|_{2}^{2}\right) \mathrm{d} s \\
& \geq \frac{1}{\eta(t)} \int_{t_{0}}^{t} \eta(t) \xi(s) H(g(s))\left(\left\|\left(3 w_{x}-\psi_{x}\right)(t)-\left(3 w_{x}-\psi_{x}\right)(t-s)\right\|_{2}^{2}\right. \\
& \left.+\left\|\left(3 w_{x t}-\psi_{x t}\right)(t)-\left(3 w_{x t}-\psi_{x t}\right)(t-s)\right\|_{2}^{2}\right) \mathrm{d} s \\
& \geq \frac{\xi(t)\left(t-t_{0}\right)}{\gamma \eta(t)} \int_{t_{0}}^{t} H(\eta(t) g(s)) \frac{\gamma}{t-t_{0}}\left(\left\|\left(3 w_{x}-\psi_{x}\right)(t)-\left(3 w_{x}-\psi_{x}\right)(t-s)\right\|_{2}^{2}\right. \\
& \left.+\left\|\left(3 w_{x t}-\psi_{x t}\right)(t)-\left(3 w_{x t}-\psi_{x t}\right)(t-s)\right\|_{2}^{2}\right) \mathrm{d} s \\
& \geq \frac{\xi(t)\left(t-t_{0}\right)}{\gamma} H\left(\frac { 1 } { \eta ( t ) } \int _ { t _ { 0 } } ^ { t } \eta ( t ) g ( s ) \frac { \gamma } { t - t _ { 0 } } \left(\left\|\left(3 w_{x}-\psi_{x}\right)(t)-\left(3 w_{x}-\psi_{x}\right)(t-s)\right\|_{2}^{2}\right.\right. \\
& \left.\left.+\left\|\left(3 w_{x t}-\psi_{x t}\right)(t)-\left(3 w_{x t}-\psi_{x t}\right)(t-s)\right\|_{2}^{2}\right) \mathrm{~d} s\right) \\
& =\frac{\xi(t)\left(t-t_{0}\right)}{\gamma} H\left(\frac { \gamma } { t - t _ { 0 } } \int _ { t _ { 0 } } ^ { t } g ( s ) \left(\left\|\left(3 w_{x}-\psi_{x}\right)(t)-\left(3 w_{x}-\psi_{x}\right)(t-s)\right\|_{2}^{2}\right.\right. \\
& \left.\left.+\left\|\left(3 w_{x t}-\psi_{x t}\right)(t)-\left(3 w_{x t}-\psi_{x t}\right)(t-s)\right\|_{2}^{2}\right) \mathrm{~d} s\right) .
\end{aligned}
$$


Since $\bar{H}$ is an extension of $H$ (see Remark 2.1 (2)), we also have

$$
\begin{aligned}
\theta(t) \geq \frac{\xi(t)\left(t-t_{0}\right)}{\gamma} \bar{H}\left(\frac { \gamma } { t - t _ { 0 } } \int _ { t _ { 0 } } ^ { t } g ( s ) \left(\left\|\left(3 w_{x}-\psi_{x}\right)(t)-\left(3 w_{x}-\psi_{x}\right)(t-s)\right\|_{2}^{2}\right.\right. \\
\left.\left.\quad+\left\|\left(3 w_{x t}-\psi_{x t}\right)(t)-\left(3 w_{x t}-\psi_{x t}\right)(t-s)\right\|_{2}^{2}\right) \mathrm{~d} s\right) .
\end{aligned}
$$

In this way,

$$
\begin{aligned}
\int_{t_{0}}^{t} g(s)( & \left\|\left(3 w_{x}-\psi_{x}\right)(t)-\left(3 w_{x}-\psi_{x}\right)(t-s)\right\|_{2}^{2} \\
& \left.+\left\|\left(3 w_{x t}-\psi_{x t}\right)(t)-\left(3 w_{x t}-\psi_{x t}\right)(t-s)\right\|_{2}^{2}\right) \mathrm{d} s \\
\leq & \frac{t-t_{0}}{\gamma} \bar{H}^{-1}\left(\frac{\gamma \theta(t)}{\xi(t)\left(t-t_{0}\right)}\right), \quad \forall t>t_{0} .
\end{aligned}
$$

Then, taking $\mathcal{F}_{1}(t):=\mathcal{F}(t)+c E(t)+c \widetilde{E}(t), 4.6$ becomes

$$
\begin{aligned}
\mathcal{F}_{1}^{\prime}(t) & :=\mathcal{F}^{\prime}(t)+c E^{\prime}(t)+c \widetilde{E}^{\prime}(t) \\
& \leq-m_{1} E(t)+\frac{c}{\gamma}\left(t-t_{0}\right) \bar{H}^{-1}\left(\frac{\gamma \theta(t)}{\xi(t)\left(t-t_{0}\right)}\right)+c_{5} g(t), \quad \forall t>t_{0} .
\end{aligned}
$$

Let $0<\varepsilon_{1}<r$, we define the functional $\mathcal{F}_{2}(t)$ by, for any $t>t_{0}$,

$$
\mathcal{F}_{2}(t):=\bar{H}^{\prime}\left(\frac{\varepsilon_{1}}{t-t_{0}} \frac{E(t)}{E(0)}\right) \mathcal{F}_{1}(t) .
$$

Then, recalling that $E^{\prime}(t) \leq 0, \bar{H}^{\prime}>0$ and $\bar{H}^{\prime \prime}>0$ as well as making use of estimate (4.8), we have

$$
\begin{aligned}
\mathcal{F}_{2}^{\prime}(t)= & \left(-\frac{\varepsilon_{1}}{\left(t-t_{0}\right)^{2}} \frac{E(t)}{E(0)}+\frac{\varepsilon_{1}}{t-t_{0}} \frac{E^{\prime}(t)}{E(0)}\right) \bar{H}^{\prime \prime}\left(\frac{\varepsilon_{1}}{t-t_{0}} \frac{E(t)}{E(0)}\right) \mathcal{F}_{1}(t) \\
& +\bar{H}^{\prime}\left(\frac{\varepsilon_{1}}{t-t_{0}} \frac{E(t)}{E(0)}\right) \mathcal{F}_{1}^{\prime}(t) \\
\leq & \bar{H}^{\prime}\left(\frac{\varepsilon_{1}}{t-t_{0}} \frac{E(t)}{E(0)}\right)\left(-m_{1} E(t)+\frac{c}{\gamma}\left(t-t_{0}\right) \bar{H}^{-1}\left(\frac{\gamma \theta(t)}{\xi(t)\left(t-t_{0}\right)}\right)+c_{5} g(t)\right) \\
= & -m_{1} E(t) \bar{H}^{\prime}\left(\frac{\varepsilon_{1}}{t-t_{0}} \frac{E(t)}{E(0)}\right)+c_{5} \bar{H}^{\prime}\left(\frac{\varepsilon_{1}}{t-t_{0}} \frac{E(t)}{E(0)}\right) g(t) \\
& +\frac{c}{\gamma}\left(t-t_{0}\right) \bar{H}^{\prime}\left(\frac{\varepsilon_{1}}{t-t_{0}} \frac{E(t)}{E(0)}\right) \bar{H}^{-1}\left(\frac{\gamma \theta(t)}{\xi(t)\left(t-t_{0}\right)}\right), \quad \forall t>t_{0} .
\end{aligned}
$$

We give the following generalized Young inequality

$$
A B \leq \bar{H}^{*}(A)+\bar{H}(B)
$$

where $\bar{H}^{*}$ is the convex conjugate of $\bar{H}$ in the sense of Young 2], i.e.,

$$
\bar{H}^{*}(s)=s\left(\bar{H}^{\prime}\right)^{-1}(s)-\bar{H}\left[\left(\bar{H}^{\prime}\right)^{-1}(s)\right] .
$$


Choosing $A=\bar{H}^{\prime}\left(\frac{\varepsilon_{1}}{t-t_{0}} \frac{E(t)}{E(0)}\right), B=\bar{H}^{-1}\left(\frac{\gamma \theta(t)}{\xi(t)\left(t-t_{0}\right)}\right)$ and combining 4.9) and 4.10, we obtain

$$
\begin{aligned}
& \bar{H}^{\prime}\left(\frac{\varepsilon_{1}}{t-t_{0}} \frac{E(t)}{E(0)}\right) \bar{H}^{-1}\left(\frac{\gamma \theta(t)}{\xi(t)\left(t-t_{0}\right)}\right) \\
\leq & \bar{H}^{*}\left(\bar{H}^{\prime}\left(\frac{\varepsilon_{1}}{t-t_{0}} \frac{E(t)}{E(0)}\right)\right)+\bar{H}\left(\bar{H}^{-1}\left(\frac{\gamma \theta(t)}{\xi(t)\left(t-t_{0}\right)}\right)\right) \\
= & \bar{H}^{*}\left(\bar{H}^{\prime}\left(\frac{\varepsilon_{1}}{t-t_{0}} \frac{E(t)}{E(0)}\right)\right)+\frac{\gamma \theta(t)}{\xi(t)\left(t-t_{0}\right)}
\end{aligned}
$$

and

$$
\begin{aligned}
\bar{H}^{*}\left(\bar{H}^{\prime}\left(\frac{\varepsilon_{1}}{t-t_{0}} \frac{E(t)}{E(0)}\right)\right)= & \bar{H}^{\prime}\left(\frac{\varepsilon_{1}}{t-t_{0}} \frac{E(t)}{E(0)}\right)\left(\bar{H}^{\prime}\right)^{-1}\left(\bar{H}^{\prime}\left(\frac{\varepsilon_{1}}{t-t_{0}} \frac{E(t)}{E(0)}\right)\right) \\
& -\bar{H}\left[\left(\bar{H}^{\prime}\right)^{-1}\left(\bar{H}^{\prime}\left(\frac{\varepsilon_{1}}{t-t_{0}} \frac{E(t)}{E(0)}\right)\right)\right] \\
= & \frac{\varepsilon_{1}}{t-t_{0}} \frac{E(t)}{E(0)} \bar{H}^{\prime}\left(\frac{\varepsilon_{1}}{t-t_{0}} \frac{E(t)}{E(0)}\right)-\bar{H}\left(\frac{\varepsilon_{1}}{t-t_{0}} \frac{E(t)}{E(0)}\right) \\
\leq & \frac{\varepsilon_{1}}{t-t_{0}} \frac{E(t)}{E(0)} \bar{H}^{\prime}\left(\frac{\varepsilon_{1}}{t-t_{0}} \frac{E(t)}{E(0)}\right) .
\end{aligned}
$$

So, combining (4.9), 4.11) and (4.12), we obtain

$$
\mathcal{F}_{2}^{\prime}(t) \leq-\left(m_{1} E(0)-c \varepsilon_{1}\right) \frac{E(t)}{E(0)} \bar{H}^{\prime}\left(\frac{\varepsilon_{1}}{t-t_{0}} \frac{E(t)}{E(0)}\right)+c_{5} \bar{H}^{\prime}\left(\frac{\varepsilon_{1}}{t-t_{0}} \frac{E(t)}{E(0)}\right) g(t)+c \frac{\theta(t)}{\xi(t)} .
$$

Then, if we fix $\varepsilon_{1}$ much smaller (if needed), we will arrive at, for all $t>t_{0}$ and $m_{2}>0$,

$$
\mathcal{F}_{2}^{\prime}(t) \leq-m_{2} \frac{E(t)}{E(0)} \bar{H}^{\prime}\left(\frac{\varepsilon_{1}}{t-t_{0}} \frac{E(t)}{E(0)}\right)+c_{5} \bar{H}^{\prime}\left(\frac{\varepsilon_{1}}{t-t_{0}} \frac{E(t)}{E(0)}\right) g(t)+c \frac{\theta(t)}{\xi(t)} .
$$

From this, we multiply 4.13 by $\xi(t)$ to get

$$
\xi(t) \mathcal{F}_{2}^{\prime}(t) \leq-m_{2} \xi(t) \frac{E(t)}{E(0)} \bar{H}^{\prime}\left(\frac{\varepsilon_{1}}{t-t_{0}} \frac{E(t)}{E(0)}\right)+c_{5} \xi(t) \bar{H}^{\prime}\left(\frac{\varepsilon_{1}}{t-t_{0}} \frac{E(t)}{E(0)}\right) g(t)+c \theta(t) .
$$

Then, using the fact that, as $\varepsilon_{1} \frac{E(t)}{E(0)}<r, \bar{H}^{\prime}\left(\frac{\varepsilon_{1}}{t-t_{0}} \frac{E(t)}{E(0)}\right)=H^{\prime}\left(\frac{\varepsilon_{1}}{t-t_{0}} \frac{E(t)}{E(0)}\right)$ and inequality (4.7) to get, for $c_{7}>0$,

$$
\begin{aligned}
\xi(t) \mathcal{F}_{2}^{\prime}(t) \leq & -m_{2} \xi(t) \frac{E(t)}{E(0)} H^{\prime}\left(\frac{\varepsilon_{1}}{t-t_{0}} \frac{E(t)}{E(0)}\right)+c_{5} \xi(t) H^{\prime}\left(\frac{\varepsilon_{1}}{t-t_{0}} \frac{E(t)}{E(0)}\right) g(t) \\
& -c\left(E^{\prime}(t)+\widetilde{E}^{\prime}(t)\right)+c_{7} g(t) .
\end{aligned}
$$

Since $1 /\left(t-t_{0}\right) \rightarrow 0$ when $t \rightarrow \infty$, there exists $t_{1}>t_{0}$ such that $1 /\left(t-t_{0}\right)<1$, whenever $t>t_{1}$. According to the strictly increasing property of $H^{\prime}$, non-increasing properties of $E$ and $\xi$, we conclude that

$$
\xi(t) H^{\prime}\left(\frac{\varepsilon_{1}}{t-t_{0}} \frac{E(t)}{E(0)}\right) \leq H^{\prime}\left(\varepsilon_{1}\right) \xi(0), \quad \forall t>t_{1} .
$$


Therefore, it is sufficient to prove that, for some positive constant $c_{8}$,

$$
\begin{aligned}
\xi(t) \mathcal{F}_{2}^{\prime}(t)+c\left(E^{\prime}(t)+\widetilde{E}^{\prime}(t)\right) & \leq-m_{2} \xi(t) \frac{E(t)}{E(0)} H^{\prime}\left(\frac{\varepsilon_{1}}{t-t_{0}} \frac{E(t)}{E(0)}\right)+c_{5} H^{\prime}\left(\varepsilon_{1}\right) \xi(0)+c_{7} g(t) \\
& \leq-m_{2} \xi(t) \frac{E(t)}{E(0)} H^{\prime}\left(\frac{\varepsilon_{1}}{t-t_{0}} \frac{E(t)}{E(0)}\right)+c_{8} g(t) .
\end{aligned}
$$

After that, defining $\mathcal{F}_{3}(t)=\xi \mathcal{F}_{2}(t)+c(E(t)+\widetilde{E}(t))$ and using the non-increasing property of $\xi(t)$, we arrive at, for any $t>t_{1}$,

$$
m_{2} \xi(t) \frac{E(t)}{E(0)} H^{\prime}\left(\frac{\varepsilon_{1}}{t-t_{0}} \frac{E(t)}{E(0)}\right) \leq-\mathcal{F}_{3}^{\prime}(t)+c_{8} g(t)
$$

Moreover, it suffices to show that the map $t \mapsto E(t) H^{\prime}\left(\frac{\varepsilon_{1}}{t-t_{0}} \frac{E(t)}{E(0)}\right)$ is non-increasing by the non-increasing property of $E(t)$ and $H^{\prime \prime}>0$. Consequently, we can take an integration of 4.14 over $\left(t_{1}, t\right)$ yields

$$
m_{2} \int_{t_{1}}^{t} \xi(s) \mathrm{d} s \frac{E(t)}{E(0)} H^{\prime}\left(\frac{\varepsilon_{1}}{t-t_{0}} \frac{E(t)}{E(0)}\right) \leq \mathcal{F}_{3}\left(t_{1}\right)+c_{8}(D-l) .
$$

If we multiply the above inequality by $1 /\left(t-t_{0}\right)>0$, for any $t>t_{1}$, we will obtain

$$
m_{2} \frac{1}{t-t_{0}} \int_{t_{1}}^{t} \xi(s) \mathrm{d} s \frac{E(t)}{E(0)} H^{\prime}\left(\frac{\varepsilon_{1}}{t-t_{0}} \frac{E(t)}{E(0)}\right) \leq \frac{\mathcal{F}_{3}\left(t_{1}\right)+c_{8}(D-l)}{t-t_{0}} .
$$

Finally, define

$$
H_{2}(t)=t H^{\prime}(t)
$$

It is immediate that $H_{2}^{\prime}(t), H_{2}(t)>0$ on $(0, r]$ by the strict convexity of $H$ on $(0, r]$. Therefore, we obtain, for two positive constants $k_{1}$ and $k_{2}$,

$$
E(t) \leq k_{2}\left(t-t_{0}\right) H_{2}^{-1}\left(\frac{k_{1}}{\left(t-t_{0}\right) \int_{t_{1}}^{t} \xi(s) \mathrm{d} s}\right), \quad \forall t>t_{1} .
$$

This completes the proof.

5. The case without structural damping under equal wave speeds

In this section, we will take account of the general decay for system $11.1-1.3$ without structural damping $(\beta=0)$ in the case of equal speeds of wave propagation.

To overcome the absence of structural damping $(\beta=0)$, we need to construct a new perturbed functional to estimate $\left\|w_{t}\right\|_{2}^{2}$. As in 18, we consider the functional

$$
J(t)=-I_{\rho} \int_{0}^{1} w_{t}\left(\psi-\varphi_{x}\right) \mathrm{d} x+I_{\rho} \int_{0}^{1} \varphi_{t} w_{x} \mathrm{~d} x .
$$

Then the following result holds: 
Lemma 5.1. Let $U$ be the solution of $(1.1)-(1.3)$. The functional $J(t)$ satisfies the estimate

$$
J^{\prime}(t) \leq-\frac{3 I_{\rho}}{2}\left\|w_{t}\right\|_{2}^{2}+\frac{2 I_{\rho}}{3}\left\|(3 w-\psi)_{t}\right\|_{2}^{2}+\frac{4 \gamma \varepsilon_{6}}{3}\|w\|_{2}^{2}+\left(G+\frac{4 \gamma}{3 \varepsilon_{6}}\right)\left\|\psi-\varphi_{x}\right\|_{2}^{2},
$$

where $\varepsilon_{6}>0$.

Proof. Differentiating $J(t)$ with respect to $t$, using (1.1) and integrating by parts, we obtain

$$
\begin{aligned}
J^{\prime}(t) & =-I_{\rho} \int_{0}^{1} w_{t t}\left(\psi-\varphi_{x}\right) \mathrm{d} x-I_{\rho} \int_{0}^{1} w_{t}\left(\psi_{t}-\varphi_{x t}\right) \mathrm{d} x+I_{\rho} \int_{0}^{1} \varphi_{t t} w_{x} \mathrm{~d} x+I_{\rho} \int_{0}^{1} \varphi_{t} w_{x t} \mathrm{~d} x \\
& =G\left\|\psi-\varphi_{x}\right\|_{2}^{2}+\frac{4 \gamma}{3} \int_{0}^{1} w\left(\psi-\varphi_{x}\right) \mathrm{d} x+\left(D-\frac{I_{\rho} G}{\rho}\right) \int_{0}^{1} w_{x}\left(\psi-\varphi_{x}\right) \mathrm{d} x-I_{\rho} \int_{0}^{1} w_{t} \psi_{t} \mathrm{~d} x .
\end{aligned}
$$

Using $\psi_{t}=3 w_{t}-(3 w-\psi)_{t}$, Young's inequality and $G / \rho=D / I_{\rho}$, we know that, for some $\varepsilon_{6}>0$

$$
\begin{aligned}
J^{\prime}(t)= & -3 I_{\rho}\left\|w_{t}\right\|_{2}^{2}+I_{\rho} \int_{0}^{1} w_{t}(3 w-\psi)_{t} \mathrm{~d} x+G\left\|\psi-\varphi_{x}\right\|_{2}^{2}+\frac{4 \gamma}{3} \int_{0}^{1} w\left(\psi-\varphi_{x}\right) \mathrm{d} x \\
& +\left(D-\frac{I_{\rho} G}{\rho}\right) \int_{0}^{1} w_{x}\left(\psi-\varphi_{x}\right) \mathrm{d} x \\
\leq & -3 I_{\rho}\left\|w_{t}\right\|_{2}^{2}+\frac{3 I_{\rho}}{2}\left\|w_{t}\right\|_{2}^{2}+\frac{2 I_{\rho}}{3}\left\|(3 w-\psi)_{t}\right\|_{2}^{2}+G\left\|\psi-\varphi_{x}\right\|_{2}^{2} \\
& +\frac{4 \gamma}{3} \varepsilon_{6}\|w\|_{2}^{2}+\frac{4 \gamma}{3 \varepsilon_{6}}\left\|\psi-\varphi_{x}\right\|_{2}^{2} \\
= & -\frac{3 I_{\rho}}{2}\left\|w_{t}\right\|_{2}^{2}+\frac{2 I_{\rho}}{3}\left\|(3 w-\psi)_{t}\right\|_{2}^{2}+\frac{4 \gamma \varepsilon_{6}}{3}\|w\|_{2}^{2}+\left(G+\frac{4 \gamma}{3 \varepsilon_{6}}\right)\left\|\psi-\varphi_{x}\right\|_{2}^{2} .
\end{aligned}
$$

This proof is now completed.

We define a new Lyapunov function $\mathcal{L}_{1}(t)$ :

$$
\mathcal{L}_{1}(t):=N E(t)+F_{1}(t)+N_{2} F_{2}(t)+N_{3} F_{3}(t)+N_{4} F_{4}(t)+N_{5} F_{5}(t)+N_{6} J(t),
$$

where $N, N_{2}, N_{3}, N_{4}, N_{5}, N_{6} \geq 0$ and $F_{i}(t), i=1,2,3,4,5$ remain as defined in Lemmas 3.13 .5 with the derivatives of $F_{i}(t), i=1,2,4,5$ remain the same while derivatives of $E(t)$ and $F_{3}(t)$ are given as follows:

$$
E^{\prime}(t)=-\frac{g(t)}{2}\left\|3 w_{x}-\psi_{x}\right\|_{2}^{2}+\frac{1}{2}\left(g^{\prime} \circ\left(3 w_{x}-\psi_{x}\right)\right)(t) \leq 0, \quad \forall t \geq 0
$$

and

$$
\begin{aligned}
F_{3}^{\prime}(t) & \leq I_{\rho}\left\|w_{t}\right\|_{2}^{2}+G \varepsilon_{3}\|w\|_{2}^{2}+\frac{G}{4 \varepsilon_{3}}\left\|\psi-\varphi_{x}\right\|_{2}^{2}-\frac{4 \gamma}{3}\|w\|_{2}^{2}-D\left\|w_{x}\right\|_{2}^{2} \\
& =-\left(\frac{4}{3} \gamma-G \varepsilon_{3}\right)\|w\|_{2}^{2}-D\left\|w_{x}\right\|_{2}^{2}+I_{\rho}\left\|w_{t}\right\|_{2}^{2}+\frac{G}{4 \varepsilon_{3}}\left\|\psi-\varphi_{x}\right\|_{2}^{2} .
\end{aligned}
$$

Now, we are ready to prove Theorem 2.10. 
Proof of Theorem 2.10. Taking derivative about $\mathcal{L}_{1}(t)$, using lemmas in Section 3 and $G / \rho=D / I_{\rho}$, we have

$$
\begin{aligned}
\mathcal{L}_{1}^{\prime}(t) \leq & -\left(\rho-\frac{N_{5} \varepsilon_{5} \rho}{G}(1+g(0))\right)\left\|\varphi_{t}\right\|_{2}^{2}-\left(\frac{3 N_{6} I_{\rho}}{2}-N_{3} I_{\rho}-18 N_{5} \varepsilon_{5} I_{\rho}\right)\left\|w_{t}\right\|_{2}^{2} \\
& -I_{\rho}\left(\frac{g_{1} N_{4}}{2}-N_{2}-N_{5}\left(2 \varepsilon_{5}+\frac{1}{4 \varepsilon_{5}}\right)-\frac{2 N_{6}}{3}\right)\left\|3 w_{t}-\psi_{t}\right\|_{2}^{2} \\
& -\left(N_{3}\left(\frac{4 \gamma}{3}-G \varepsilon_{3}\right)-\frac{4 N_{6} \gamma \varepsilon_{6}}{3}\right)\|w\|_{2}^{2}-\left(N_{3} D-\frac{9 G}{2 \varepsilon_{1}}\right)\left\|w_{x}\right\|_{2}^{2} \\
& -\left(N_{5} G-\varepsilon_{1} G-\frac{G N_{2}}{4 \varepsilon_{2}}-\frac{G N_{3}}{4 \varepsilon_{3}}-\frac{N_{4} \varepsilon_{4} G}{2}-N_{6}\left(G+\frac{4 \gamma}{3 \varepsilon_{6}}\right)\right)\left\|\psi-\varphi_{x}\right\|_{2}^{2} \\
& -\left(g(t)\left(\frac{N}{2}-\frac{N_{5} \rho}{4 \varepsilon_{5} G}\right)+N_{2}\left(l-\varepsilon_{2}(G+1)\right)-\frac{G}{2 \varepsilon_{1}}-N_{4} \varepsilon_{4} \frac{D}{2}\right)\left\|3 w_{x}-\psi_{x}\right\|_{2}^{2} \\
& +(D-l)\left(\frac{N_{2}}{4 \varepsilon_{2}}+N_{4}\left(\frac{G \lambda_{0}}{2 \varepsilon_{4}}+\frac{D}{2 \varepsilon_{4}}\right)\right)\left(g \circ\left(3 w_{x}-\psi_{x}\right)\right)(t) \\
& +\left(\frac{N}{2}-\frac{N_{4} I_{\rho} g(0) \lambda_{0}}{2 g_{0}(t)}-\frac{N_{5} \rho g(0)}{4 \varepsilon_{5} G}\right)\left(g^{\prime} \circ\left(3 w_{x}-\psi_{x}\right)\right)(t) .
\end{aligned}
$$

At this point, we need to choose our constants very carefully. First, we choose

$$
\varepsilon_{1}=G, \quad \varepsilon_{2}=\frac{1}{N_{2}}, \quad \varepsilon_{3}=\frac{1}{N_{3}}, \quad \varepsilon_{4}=\frac{1}{N_{4}}, \quad \varepsilon_{5}=\frac{G}{2 N_{5}(1+g(0))}, \quad \varepsilon_{6}=\frac{1}{N_{6}},
$$

so that

$$
\begin{aligned}
\mathcal{L}_{1}^{\prime}(t) \leq & -\frac{\rho}{2}\left\|\varphi_{t}\right\|_{2}^{2}-I_{\rho}\left(\frac{N_{4} g_{1}}{2}-N_{2}-\frac{N_{5}^{2}(1+g(0))}{2 G}-\frac{G}{1+g(0)}-\frac{2 N_{6}}{3}\right)\left\|3 w_{t}-\psi_{t}\right\|_{2}^{2} \\
& -\left(N_{3} D-\frac{9}{2}\right)\left\|w_{x}\right\|_{2}^{2}-\left(\frac{3 N_{6} I_{\rho}}{2}-N_{3} I_{\rho}-\frac{9 I_{\rho} G}{1+g(0)}\right)\left\|w_{t}\right\|_{2}^{2} \\
& -\left(\frac{4 N_{3} \gamma}{3}-\left(G+\frac{4 \gamma}{3}\right)\right)\|w\|_{2}^{2} \\
& -\left(N_{5} G-\frac{G N_{2}^{2}}{4}-\frac{G N_{3}^{2}}{4}-\frac{G}{2}-G^{2}-N_{6} G-\frac{4 \gamma N_{6}^{2}}{3}\right)\left\|\psi-\varphi_{x}\right\|_{2}^{2} \\
& -\left(g(t)\left(\frac{N}{2}-\frac{N_{5}^{2} \rho(1+g(0))}{2 G^{2}}\right)+N_{2} l-\left(\frac{3}{2}+G+\frac{D}{2}\right)\right)\left\|3 w_{x}-\psi_{x}\right\|_{2}^{2} \\
& +(D-l)\left(\frac{N_{2}^{2}}{4}+N_{4}^{2}\left(\frac{G \lambda_{0}}{2}+\frac{D}{2}\right)\right)\left(g \circ\left(3 w_{x}-\psi_{x}\right)\right)(t) \\
& +\left(\frac{N}{2}-\frac{N_{4} I_{\rho} g(0) \lambda_{0}}{2 g_{0}(t)}-\frac{N_{5}^{2} \rho(1+g(0))}{2 G^{2}}\right)\left(g^{\prime} \circ\left(3 w_{x}-\psi_{x}\right)\right)(t) .
\end{aligned}
$$

Next, we select $N_{2}$ large enough so that

$$
N_{2} l-\left(\frac{3}{2}+G+\frac{D}{2}\right)>0 .
$$


Then, we choose $N_{3}$ large enough so that

$$
N_{3} D-\frac{9}{2}>0 \quad \text { and } \quad \frac{4 N_{3} \gamma}{3}-\left(G+\frac{4 \gamma}{3}\right)>0 .
$$

Furthermore, we select $N_{6}$ large enough so that

$$
\frac{3 N_{6} I_{\rho}}{2}-N_{3} I_{\rho}-\frac{9 I_{\rho} G}{1+g(0)}>0 .
$$

After that, we select $N_{5}$ large enough so that

$$
N_{5} G-\frac{G N_{2}^{2}}{4}-\frac{G N_{3}^{2}}{4}-\frac{G}{2}-G^{2}-N_{6} G-\frac{4 \gamma N_{6}^{2}}{3}>0 .
$$

Then, we select $N_{4}$ large enough so that

$$
I_{\rho}\left(\frac{N_{4} g_{1}}{2}-N_{2}-\frac{N_{5}^{2}(1+g(0))}{2 G}-\frac{G}{1+g(0)}-\frac{2 N_{6}}{3}\right)>0 .
$$

Finally, we choose $N$ large enough so that

$$
\frac{N}{2}-\frac{N_{5}^{2} \rho(1+g(0))}{2 G^{2}}>0 \text { and } \quad \frac{N}{2}-\frac{N_{4} I_{\rho} g(0) \lambda_{0}}{2 g_{0}(t)}-\frac{N_{5}^{2} \rho(1+g(0))}{2 G^{2}}>0 .
$$

Combining (5.1) and the above, we deduce that (5.1) becomes, for positive constants $C_{i}$, $i=2,3,4$,

$$
\begin{aligned}
\mathcal{L}_{1}^{\prime}(t) \leq & -C_{2}\left[\left\|\varphi_{t}\right\|_{2}^{2}+\left\|3 w_{t}-\psi_{t}\right\|_{2}^{2}+\left\|w_{x}\right\|_{2}^{2}+\left\|w_{t}\right\|_{2}^{2}+\|w\|_{2}^{2}+\left\|\psi-\varphi_{x}\right\|_{2}^{2}+\left\|3 w_{x}-\psi_{x}\right\|_{2}^{2}\right] \\
& +C_{3}\left(g \circ\left(3 w_{x}-\psi_{x}\right)\right)(t)+C_{4}\left(g^{\prime} \circ\left(3 w_{x}-\psi_{x}\right)\right)(t) \\
\leq & -C_{2}\left[\left\|\varphi_{t}\right\|_{2}^{2}+\left\|3 w_{t}-\psi_{t}\right\|_{2}^{2}+\left\|w_{x}\right\|_{2}^{2}+\left\|w_{t}\right\|_{2}^{2}+\|w\|_{2}^{2}+\left\|\psi-\left.\varphi_{x}\right|_{2} ^{2}+\right\| 3 w_{x}-\psi_{x} \|_{2}^{2}\right] \\
& +C_{3}\left(g \circ\left(3 w_{x}-\psi_{x}\right)\right)(t) \\
\leq & -m E(t)-c E^{\prime}(t)+c \int_{0}^{1} \int_{t_{0}}^{t} g(s)\left[\left(3 w_{x}-\psi_{x}\right)(t)-\left(3 w_{x}-\psi_{x}\right)(t-s)\right]^{2} \mathrm{~d} s \mathrm{~d} x .
\end{aligned}
$$

The remainder of the proof is similar to that in the proof of Theorem 2.7 (see [22, 23]). This completes the proof of Theorem 2.10 .

\section{Acknowledgments}

This work was supported by the National Natural Science Foundation of China (grant number 11771216), the Natural Science Foundation of Jiangsu Province (grant number BK20151523), the Six Talent Peaks Project in Jiangsu Province (grant number 2015-XCL020) and the Qing Lan Project of Jiangsu Province. 


\section{References}

[1] T. A. Apalara, Uniform stability of a laminated beam with structural damping and second sound, Z. Angew. Math. Phys. 68 (2017), no. 2, Art. 41, 16 pp.

[2] V. I. Arnol'd, Mathematical Methods of Classical Mechanics, Graduate Texts in Mathematics 60, Springer-Verlag, New York, 1989.

[3] J. Bajkowski, J. R. Fernández, K. L. Kuttler and M. Shillor, A thermoviscoelastic beam model for brakes, European J. Appl. Math. 15 (2004), no. 2, 181-202.

[4] F. Boulanouar and S. Drabla, General boundary stabilization result of memory-type thermoelasticity with second sound, Electron. J. Differential Equations 2014 (2014), no. 202,18 pp.

[5] A. D. S. Campelo, D. S. Almeida Júnior and M. L. Santos, Stability to the dissipative Reissner-Mindlin-Timoshenko acting on displacement equation, European J. Appl. Math. 27 (2016), no. 2, 157-193.

[6] X.-G. Cao, D.-Y. Liu and G.-Q. Xu, Easy test for stability of laminated beams with structural damping and boundary feedback controls, J. Dyn. Control Syst. 13 (2007), no. $3,313-336$.

[7] M. M. Cavalcanti and A. Guesmia, General decay rates of solutions to a nonlinear wave equation with boundary condition of memory type, Differential Integral Equations 18 (2005), no. 5, 583-600.

[8] M. Chen, W. Liu and W. Zhou, Existence and general stabilization of the Timoshenko system of thermo-viscoelasticity of type III with frictional damping and delay terms, Adv. Nonlinear Anal. 7 (2018), no. 4, 547-569.

[9] B. Feng, On a semilinear Timoshenko-Coleman-Gurtin system: quasi-stability and attractors, Discrete Contin. Dyn. Syst. 37 (2017), no. 9, 4729-4751.

[10] _ Well-posedness and exponential decay for laminated Timoshenko beams with time delays and boundary feedbacks, Math. Methods Appl. Sci. 41 (2018), no. 3, 1162-1174.

[11] S. W. Hansen, A model for a two-layered plate with interfacial slip, in: Control and Estimation of Distributed Parameter Systems: Nonlinear Phenomena (Vorau, 1993), 143-170, Internat. Ser. Numer. Math. 118, Birkhäuser, Basel, 1994.

[12] S. W. Hansen and R. D. Spies, Structural damping in laminated beams due to interfacial slip, J. Sound Vibration 204 (1997), no. 2, 183-202. 
[13] G. Li, X. Kong and W. Liu, General decay for a laminated beam with structural damping and memory: the case of non-equal wave speeds, J. Integral Equations Appl. 30 (2018), no. 1, 95-116.

[14] W. Liu, K. Chen and J. Yu, Existence and general decay for the full von Kármán beam with a thermo-viscoelastic damping, frictional dampings and a delay term, IMA J. Math. Control Inform. 34 (2017), no. 2, 521-542.

[15] _ Asymptotic stability for a non-autonomous full von Kármán beam with thermo-viscoelastic damping, Appl. Anal. 97 (2018), no. 3, 400-414.

[16] W. Liu, Y. Sun and G. Li, On decay and blow-up of solutions for a singular nonlocal viscoelastic problem with a nonlinear source term, Topol. Methods Nonlinear Anal. 49 (2017), no. 1, 299-323.

[17] W. Liu, J. Yu and G. Li, Exponential stability of a flexible structure with second sound, Ann. Polon. Math., in press. doi: 10.4064/ap171116-31-8

[18] W. Liu and W. Zhao, Stabilization of a thermoelastic laminated beam with past history, Appl. Math. Optim., in press. doi: 10.1007/s00245-017-9460-y

[19] W. Liu, B. Zhu, G. Li and D. Wang, General decay for a viscoelastic Kirchhoff equation with Balakrishnan-Taylor damping, dynamic boundary conditions and a timevarying delay term, Evol. Equ. Control Theory 6 (2017), no. 2, 239-260.

[20] A. Lo and N.-e. Tatar, Uniform stability of a laminated beam with structural memory, Qual. Theory Dyn. Syst. 15 (2016), no. 2, 517-540.

[21] S. A. Messaoudi and J. H. Hassan, New general decay results for a viscoelastic-type Timoshenko system, preprint.

[22] M. I. Mustafa, General decay result for nonlinear viscoelastic equations, J. Math. Anal. Appl. 457 (2018), no. 1, 134-152.

[23] _ Laminated Timoshenko beams with viscoelastic damping, J. Math. Anal. Appl. 466 (2018), no. 1, 619-641.

[24] Y. Qin, X.-G. Yang and Z. Ma, Global existence of solutions for the thermoelastic Bresse system, Commun. Pure Appl. Anal. 13 (2014), no. 4, 1395-1406.

[25] C. A. Raposo, Exponential stability for a structure with interfacial slip and frictional damping, Appl. Math. Lett. 53 (2016), 85-91. 
[26] F. Tahamtani and A. Peyravi, Asymptotic behavior and blow-up of solutions for a nonlinear viscoelastic wave equation with boundary dissipation, Taiwanese J. Math. 17 (2013), no. 6, 1921-1943.

[27] J.-M. Wang, G.-Q. Xu and S.-P. Yung, Exponential stabilization of laminated beams with structural damping and boundary feedback controls, SIAM J. Control Optim. 44 (2005), no. 5, 1575-1597.

[28] B. Wu and J. Liu, Determination of an unknown source for a thermoelastic system with a memory effect, Inverse Problems 28 (2012), no. 9, 095012, 17 pp.

[29] B. Wu, S. Wu, J. Yu and Z. Wang, Determining the memory kernel from a fixed point measurement data for a parabolic equation with memory effect, Comput. Appl. Math. 37 (2018), no. 2, 1877-1893.

Zhijing Chen, Wenjun Liu and Dongqin Chen

College of Mathematics and Statistics, Nanjing University of Information Science and Technology, Nanjing 210044, China

E-mail address: zhijingchen@139.com, wjliu@nuist.edu.cn, cdqlinsey@163.com 